\title{
Observer ses pairs lors de séances de simulation haute-fidélité : une activité de nature ludique portée par des en-jeux sérieux
}

Obseving peers during high-fidelity simulation sessions: a playful activity by nature, driven by serious stakes

Hélène Bouchot et Serge Leblanc

\section{(2) OpenEdition Journals}

Édition électronique

URL : http://journals.openedition.org/activites/4425

DOI : $10.4000 /$ activites. 4425

ISSN : $1765-2723$

Éditeur

ARPACT - Association Recherches et Pratiques sur les ACTivités

\section{Référence électronique}

Hélène Bouchot et Serge Leblanc, «Observer ses pairs lors de séances de simulation haute-fidélité : une activité de nature ludique portée par des en-jeux sérieux », Activités [En ligne], 16-2 | 2019, mis en ligne le 15 octobre 2019, consulté le 17 octobre 2019. URL : http://journals.openedition.org/activites/ 4425 ; DOI : 10.4000/activites.4425

Ce document a été généré automatiquement le 17 octobre 2019

\section{(c) $($ †) $\ominus$}

Activités est mis à disposition selon les termes de la licence Creative Commons Attribution - Pas d'Utilisation Commerciale - Pas de Modification 4.0 International. 


\title{
Observer ses pairs lors de séances de simulation haute-fidélité : une activité de nature ludique portée par des en-jeux sérieux
}

\author{
Obseving peers during high-fidelity simulation sessions: a playful activity by
} nature, driven by serious stakes

Hélène Bouchot et Serge Leblanc

\section{NOTE DE L'ÉDITEUR}

Article soumis le 16 décembre 2018, accepté le 04 septembre 2019

\section{Introduction}

L'observation lors de séance de simulation haute-fidélité (SHF) est une pratique ordinaire en formation initiale des métiers de la santé. Elle permet de répondre à la contrainte de former un effectif conséquent d'étudiants à travers un dispositif pédagogique qui ne soit pas trop chronophage. L'incitation forte, de la Haute Autorité de Santé (HAS, 2012a, 2017) et de la Direction Générale des Offres de Soins (DGOS, 2013), d'implémenter la SHF dans les curriculums de formation, participe à une mise en tension entre les diverses modalités d'apprentissages disciplinaires. La conception de séances de SHF par groupe d'étudiants est un levier permettant de réduire cette tension. L'équipement technologique des plateformes de SHF, notamment les dispositifs vidéo préconisés par la HAS, favorise l'instauration de séances alternant activité simulée et activité d'observation de pairs. Cette activité d'observation n'est pas questionnée par la HAS, dont la focale est l'activité simulée, et est peu traitée dans la littérature scientifique. Notre contribution vise la production de connaissances sur 
cette activité énigmatique, en partie silencieuse et subjective. Nous postulons qu'analyser l'activité d'observation contribuera à repérer et identifier le potentiel d'apprentissage-développement de cet espace annexé systématiquement au dispositif qui constitue, aujourd'hui, un point aveugle de la formation des sages-femmes. Dans une première partie, nous décrivons la conduite type d'une séance de SHF en mettant en évidence le peu de références et l'absence de recommandations relatives à l'activité d'observation. Dans une deuxième partie, nous montrons que les quelques recherches relatives à l'observation de situations de SHF ne permettent pas de comprendre ce qui constitue cette activité. Cela nous conduit à élargir notre exploration à d'autres domaines professionnels (celui de la formation des enseignants) et à nous appuyer sur les résultats de contributions scientifiques, relatives à l'activité d'observation médiée par des dispositifs de vidéoformation. Dans une troisième partie, nous présentons les concepts-clés retenus dans le programme de recherche du cours d'action (Theureau, 2015) dans lequel s'inscrit notre recherche ainsi que l'observatoire mis en place auprès de cinq étudiantes sages-femmes, à l'occasion d'un module constitué de cinq séances de SHF nommé urgences obstétrico-pédiatiques. Enfin, dans une quatrième partie, nous montrons, à partir de la modélisation des cours d'expériences des étudiantes, comment l'activité s'organise collégialement en conduite de jeu et comment elle s'accompagne de processus transformatifs majorants.

\section{L'observation, une activité peu évoquée dans les prescriptions institutionnelles de formation par SHF en santé}

\subsection{La formation par SHF en santé}

2 Former par simulation en santé, participe à améliorer la qualité des soins, la culture de sécurité et respecte une démarche éthique de l'apprentissage limitant toute première expérience sur un patient (DGOS, 2013 ; HAS, 2012b). La SHF ${ }^{1}$ consiste en la construction d'une situation analogue au réel. Cet environnement simulé participe à instrumenter (Rabardel, 1995) une activité constitutive d'acquisitions, de renforcements ou de transformations de compétences techniques (hard skills) et non techniques (Soft skills) dont les «crisis ressources management» (Jaffrelot, Boet, Di Cioccio, Michinov, \& Chiniara, 2013). C'est un moyen de s'entrainer à agir en situation-problème, de créer de l'expérience en situation extraordinaire, d'analyser des pratiques situées. Afin d'accompagner et encourager son développement en formation initiale et continue, des métiers de la santé, la HAS a balisé, par ses recommandations la conception et conduite d'une séance, les principes d'évaluation des formations et les modalités de qualifications et d'évaluation des formateurs (HAS, 2012a, 2017).

3 Le déroulé type d'une séance de SHF se compose de quatre temps (HAS, 2012a).

\section{Le briefing général}

4 Il permet de familiariser les apprenants avec l'outil et les place dans des conditions psychologiques et affectives propices à l'engagement dans l'activité simulée (Dieckmann, Molin Friis, Lippert, \& Østergaard, 2009).

\section{Le pré briefing}


Il initie l'histoire de la situation expérientielle et prépare l'immersion des acteurs dans la situation simulée.

\section{La situation de simulation}

6 Les acteurs y déploient une activité qui est dynamique, contrainte par un espace-temps pour produire une expérience au plus près du réel. La scénarisation de la situation et les divers artefacts ${ }^{2}$ placés dans l'environnement, génèrent une réalité virtuelle qui rend possibles diverses interactions (hommes-hommes-artefacts) et favorise le « faire comme si » (Horcik \& Durand, 2015). Lors de formation en groupe, les apprenants nonparticipants sont observateurs, en direct, de la situation de simulation. Un dispositif technique audiovisuel permet «d'observer sans perturber les apprenants » en salle de débriefing (HAS, 2012a, p. 66).

\section{Le débriefing}

7 Il se réalise dès la fin de la situation de simulation. C'est le temps pédagogique considéré comme incontournable en simulation (Boet, Granry, \& Savoldelli, 2013). Il débute par un temps de description du vécu des acteurs. Cette étape permet de poser les émotions et facilite les échanges ultérieurs. Suit une phase d'analyse qui s'intéresse à reconstruire la cohérence donnée par les acteurs aux actions menées. Les échanges collégiaux contribuent à discuter de stratégies alternatives pertinentes dans la situation concernée. Le débriefing est un temps d'apprentissage collectif, centré sur l'activité simulée. Les interactions entre pairs et /ou experts sont considérées comme la clé de voute du développement cognitif (Dieckmann et al., 2009). Le débriefing se clôture par un temps de synthèse, avec décontextualisation des apprentissages.

8 Le postulat pédagogique de cette modalité de formation est la transférabilité des compétences développées dans et sur l'activité simulée, en pratique réelle (Boet et al. , 2013).

\subsection{La place de l'observation en SHF dans les textes institutionnels et scientifiques}

9 L'activité d'observation lors de séances de SHF est peu abordée dans les préconisations de bonnes pratiques à l'usage des formateurs de la HAS. Les références ${ }^{3}$ effectuées laissent supposer que l'observation est peu constitutive d'apprentissage en référence à l'activité simulée et que son potentiel transformatif s'actualise uniquement lors du débriefing de l'activité observée «la vidéo permet aux apprenants "non participants" de pouvoir observer la retransmission du scénario et ainsi de pouvoir bénéficier au mieux du débriefing» (HAS, 2012a, p.16). Par ailleurs, l'implication dans l'activité d'observation n'est pas jugée certaine comme le laisse penser cet extrait : « répartir les rôles d'observateurs: tous les participants restants doivent être impliqués » (HAS, 2012a, p. 54). Aucune préconisation sur l'accompagnement de cette activité, par répartition des rôles des observateurs, ne complète le modus operandi de référence.

10 Les recherches scientifiques relatives à l'observation de situation de SHF en santé restent peu nombreuses. Sur les dix dernières années, moins de 10 publications pertinentes ont pu être identifiées. Elles se situent dans le courant de recherche de "evidence based learning" ${ }^{4}$ et adoptent des méthodes quantitatives, mixtes ou qualitatives. 
11 Les études quantitatives et mixtes ont pour objectif de comparer, post débriefing, les gains de connaissances d'étudiants infirmiers ou en médecine, selon qu'ils sont observateurs ou acteurs en situation simulée. Lorsque les étudiants sont observateurs, sans aucune activité simulée lors de la formation, une grille de soutien à l'observation est instaurée. L'ensemble de ces études montre un impact positif de l'observation sur l'ensemble des variables évaluées (communication, procédure en urgence, techniques) sans différence significative entre observateurs et acteurs des situations simulées (LeBel, Haverstock, Cristancho, van Eimeren, \& Buckingham, 2018; O'Regan, Molloy, Watterson, \& Nestel, 2016 ; Zottamnn, Dieckermann, Taraswzow, Rall, \& Fischer, 2018).

Les études mixtes complètent leurs données quantitatives de données issues d'entretiens individuels afin d'accéder au vécu de quelques observateurs. Les résultats montrent des expériences à valence positive. Les observateurs rapportent se sentir impliqués dans la situation observée, que l'observation leurs permet de prendre du recul pour analyser la situation, notamment par absence de stress, que les erreurs de leurs pairs et le débriefing collégial participent à leur apprentissage (Hober \& Bonnel, 2014 ; LeBel et al., 2018).

13 L'étude qualitative adopte une approche ethnographique et s'intéresse au vécu de 10 étudiants infirmiers observateurs d'un jeu de rôle, sans grille d'observation. Les résultats construits sur la base d'entretiens individuels montrent des expériences à valence négative : manque d'implication dans l'activité, incompréhension des rôles de chacun, frustration d'être observateur. Les conclusions de l'étude pointent la nécessité d'objectiver clairement les rôles de chacun, notamment celui des observateurs et de limiter leur nombre lorsque l'observation est participante (Harder et al., 2013).

14 Une partie des résultats révèle donc des effets plus nuancés, voire négatifs, lorsque l'observation n'est pas objectivée. L'usage d'outils de soutien à l'observation en SHF avec mannequin haute-fidélité n'est pas une pratique ordinaire en France. Nous pouvons supposer que l'absence d'objectivation et de supervision de l'observation dans nos dispositifs peut engager indifféremment les étudiants dans l'activité, et interroge la portée de l'observation telle qu'elle est instituée.

Enfin les résultats des évaluations de connaissances et d'habiletés techniques ne permettent pas de distinguer les transformations qui accompagnent l'activité observation, de celles qui relèvent du remplissage de la grille de soutien ou encore de l'activité déployée lors du débriefing.

16 C'est l'enjeu de notre recherche d'éclairer ce qui se joue et se passe durant ce temps spécifique d'observation qui reste une zone d'ombre, un point aveugle à la fois du point de vue scientifique et également du point de vue de la formation. Notre étude s'intéresse donc à l'activité d'observation dans sa temporalité distincte du débriefing et selon une perspective herméneutique. Comment les étudiants s'engagent-ils dans l'acte d'observer dans cet espace du dispositif de formation SHF non prescriptif? Endossentils un rôle d'observateur, avec une implication réflexive dans la situation observée ou de spectateur, sans engagement analytique? Qu'apprennent-ils? Que ressentent-ils ? Afin de mieux circonscrire notre objet d'étude, nous nous sommes appuyés sur des recherches compréhensives portant sur l'activité d'observation de pairs, médiée par des artefacts vidéo, en situations éducatives. 


\section{Apports des approches compréhensives et situées sur l'observation en contexte de vidéoformation} situations professionnelles, proposent des environnements éducatifs qui s'apparentent au contexte d'observation de situations de SHF. La vidéo-formation est présentée comme un moyen de se décaler des enseignements théoriques pour plonger les apprenants dans les questions, problèmes et solutions inhérentes à la pratique professionnelle (Gaudin \& Chaliès, 2012).

Les problématiques de ces recherches portent sur la mise en relation de l'activité visionnée par les formés avec leur propre activité de travail et/ou les processus de transformation qui accompagnent cette activité et/ou les conditions technologiques qui favorisent les transformations de l'activité d'enseignement.

\subsection{Engagements typiques dans l'activité d'observation}

Les modélisations de l'activité des observateurs montrent que, quelles que soient les variabilités des supports de vidéo-formation, trois phénomènes typiques l'accompagnent (Flandin, Leblanc, \& Muller, 2015) :

- l'immersion: elle caractérise l'engagement dans l'activité, comme effet lié aux propriétés intrinsèques de l'artefact vidéo et à la familiarité de l'activité observée avec le vécu de l'observateur (Leblanc, 2012). L'immersion consiste pour l'observateur à occulter partiellement ou totalement sa situation présente pour y substituer la situation visionnée.

- la résonance: elle réfère à l'actualisation-synchronisation d'une préoccupation entre l'activité de l'observateur et celle de l'observé. Ce phénomène nommé «amorçage observationnel » traduit la reconnaissance de l'enjeu de la situation professionnelle de façon mimétique ou projective selon le degré de familiarité qu'elle entretient avec une situation vécue (Gaudin, Flandin, Ria, \& Chaliès, 2016).

- l'expérience fictionnelle et mimétique: elle concerne l'engagement corporel de l'observateur dans la situation visionnée (Roche \& Gal-Petitfaux, 2012). Elle traduit la projection de l'observateur à la place de l'autre. L'observateur éprouve des émotions pour l'observé et s'immerge sensoriellement. Il perçoit les matérialités spatiales, temporelles, sonores ainsi que les dimensions sociales de la communication co-produites par son interaction avec la situation visionnée. Par des processus de synchronisations, créations, attentes, anticipations, projections, l'observateur rejoue la situation comme sienne (Leblanc, 2014a). Cette expérience fictionnelle suscite des apprentissages par mimesis dans un rapport à l'action d'autres personnes que nous voyons agir et qui sont impliquées dans une scène sociale qui a du sens pour nous: «En s'appropriant mimétiquement les espaces sociaux extérieurs, les individus les transposent par les sens et l'imagination en images intérieures, en mondes du son, du toucher, de l'odorat et du goût qu'ils incorporent à leur propre monde » (Wulf, 2007, p. 11).

Lorsque l'observation est collective, les expériences interprétatives et mimétiques se doublent d'expériences interprétatives partagées, d'expériences langagières (verbalisation des expériences évoquées dans le dispositif), et d'expériences corporelles projetées dans le futur (Roche \& Gal-Petitfaux, 2012). 
Ainsi l'engagement dans l'activité et les différentes expériences qui l'accompagnent, participent à transformer l'activité directement, en lien avec la médiation vidéo (immersion, résonance, expérience fictionnelle et mimétique), ou indirectement en envisageant de nouveaux champs de possibles (processus réflexifs) (Flandin \& Ria, 2018 ; Leblanc, 2015). De ces transformations émanent de nouvelles connaissances.

\subsection{Processus de transformations typiques qui accompagnent l'activité d'observation en situation de « simulation vidéo »}

Dans un contexte de vidéoformation, l'apprentissage dépend initialement de l'actualisation d'une préoccupation puis accompagne l'identification, dans l'activité observée, d'actions contribuant au rétablissement ou à la viabilité de la situation professionnelle (Flandin \& Ria, 2018). L'apprentissage est conçu, ici, comme émergent d'un mouvement itératif d'immersion-distanciation-consistant à passer d'un point de vue égocentré (je vis ce que je vois comme si c'était moi qui le vivais), à un point de vue allocentré (je me mets à la place de l'autre) à un point de vue générique, décontextualisé (discours en 3e personne moins lié au vécu) (Ria \& Leblanc, 2011). Ce mouvement permet de déplacer l'engagement des observateurs dans la situation visionnée d'une approche compassionnelle à une approche empathique plus propice à la compréhension et à la réflexivité (Ria \& Leblanc, 2011). De ce jeu de proximitédistance, émanent des transformations des connaissances relatives à l'usage du multimédia, à sa propre pratique, aux pratiques de références, par intégration continue du nouveau au déjà-là ou par actualisation d'un potentiel caché déjà-là (Flandin et al., 2015). Les résultats des recherches montrent que la vidéo-formation permet de sensibiliser les futurs enseignants aux difficultés typiques de l'agir professionnel, de les déculpabiliser, de les rassurer et de les aider à projeter, dans des temporalités plus ou moins longues, des possibilités d'actions à tester en pratique (Leblanc, 2018). Ainsi, la vidéo-formation est un dispositif de formation qui ne peut se substituer à la pratique, mais qui la prépare (Ria, Serres, \& Leblanc, 2010).

\subsection{Potentialiser l'activité d'observation dans une visée transformative}

24 Les résultats des études, s'intéressant à la portée transformative de l'activité d'observation, médiée par le visionnage de vidéo, rapportent des variabilités de l'apprentissage majorant relatives (Gaudin, Flandin, Moussay, \& Chaliès, 2018) :

a. à la capacité qu'a l'observateur de se détacher de la situation observée tout en y restant impliqué,

b. à la capacité d'observer et analyser la pluri-dimentionnalité de la situation qui, à défaut, peut s'apparenter à une activité de butinage,

c. au degré de familiarité qu'entretiennent les situations vidéoscopées et les expériences des observateurs.

Le potentiel d'apprentissage, généré par les dispositifs vidéo, nécessite d'accompagner l'analyse réflexive pour capaciter l'attribution de significations aux évènements observés (Leblanc, 2018). Cet accompagnement devrait, selon les auteurs, consister à étayer l'articulation entre mémoire, actualité et anticipation-imagination (Leblanc, 2014b). Enfin, le choix des vidéos devrait tenir compte du niveau de développement du 
professionnel pour que ses préoccupations soient en adéquation avec le type de situation observée (Gaudin et al., 2016).

Ces résultats participent à re-questionner l'activité d'observation de situation de SHF qui n'est pas l'activité sur laquelle repose la conception du dispositif de formation, comme c'est le cas pour ces études. Comment cette observation, non objectivée, pensée comme simple moyen permettant de participer et comprendre le débriefing qui lui succède, s'organise-t-elle individuellement et collectivement lors du visionnage?

La simulation haute-fidélité est un moyen de créer de l'expérience en situations exceptionnelles, voire extraordinaires. Les situations observées n'ont peut-être jamais été expérimentées en situations réelles. Comment les observateurs s'engagent-ils dans l'activité d'observation de situations qui offrent peu de lien de familiarité avec leurs expériences antérieures? L'activité s'accompagne-t-elle des mêmes phénomènes d'immersion, résonnance et immersion mimétique? Sont-ils en capacité d'associer observation et analyse réflexive dans ce contexte formatif? Quelle est la portée transformative de l'observation qui est non accompagnée et quels types d'apprentissages concerne-t-elle?

Nous pensons que comprendre cette activité systématiquement rattachée à toute séance de SHF, en dégager ses spécificités et généralités, et identifier les processus d'apprentissage-développement, qui l'accompagnent, participe à apporter une réponse épistémique à ces questions, et à renouveler les pratiques de formation en prenant en compte sérieusement et de façon holistique, les activités qui y sont déployées.

\section{Cadre théorique de l'étude et observatoire mis en place}

Notre étude adopte le programme de recherche dit du cours d'action. Il nous donne les moyens théorico-méthodologiques de pister les transformations de l'activité via la reconstruction des cours d'action de chaque étudiante en situation d'observation.

\subsection{Cadre théorique cours d'action}

L'objet théorique cours d'action permet de reconstruire l'activité du point de vue des observatrices (dimension intrinsèque de l'activité nommée cours d'expérience) et de montrer comment cette activité donne lieu à expérience en termes de causalités et/ou conséquences inférées et constatées dans les corps, situations et cultures (Theureau, 2015). Nous souhaitons comprendre comment s'organise cette activité et identifier si elle s'accompagne d'apprentissages-développements pertinents pour la pratique maïeutique.

\section{Trois hypothèses précisent ce cadre théorique et justifient la méthodologie de} recherche (Theureau, 2006)

\section{Hypothèse 1 : l'Enaction}

Elle traduit le paradigme de l'autopoïèse ${ }^{6}$ (Varela, 1989) en termes d'activité humaine. Chaque acteur fait émerger (énacter) un monde signifiant pour lui et la dynamique de son couplage structurel avec l'environnement s'accompagne à tout moment, de 
créations ou manifestations de savoirs. Il en découle plusieurs implications spécifiant l'activité :

- elle est autonome en raison de ses propriétés d'auto-organisation. L'acteur définit ce qui est signifiant pour lui dans la situation et construit un monde qui lui est subjectivement propre, en relation avec son vécu.

- elle est située, son inscription dans un contexte spatial, matériel et social, participe à sa construction. Les composantes de l'environnement (espace, objets, dispositifs techniques, autres acteurs en présence) offrent un ensemble de ressources qui, selon leurs prises en compte par l'acteur et l'usage qu'il en fait, transforme la perception et le pouvoir d'action. Les artefacts (Norman, 1993) sont entendus comme des ressources cognitives qui capacitent l'action et les relations avec l'environnement, tout en les contraignants.

- la construction de significations, en situation, implique que l'activité s'accompagne de manifestations de connaissances pré-existantes ou construites à chaque instant dans l'action : elle est intrinsèquement cognitive.

- elle prend racine dans le corps de l'individu. La connaissance émerge de cette relation corporelle avec l'environnement.

L'activité humaine dans ce paradigme est définie comme «à la fois cognitive, autonome, incarnée, située dynamiquement dans un monde où existent d'autres acteurs, inséparablement individuelle et collective, techniquement constituée, cultivée et vécue » (Theureau, 2006, p. 40). Selon, cette hypothèse il apparait impossible, par la simple observation du comportement des étudiantes, d'accéder à leur activité.

\section{Hypothèse 2 : l'expérience}

La conscience pré réflexive (Sartre, 1943) est interprétée, selon la caractéristique de vécu de l'activité humaine, comme expérience (Theureau, 2015). L'expérience est la part attentive, éveillée, émergente de la dynamique du couplage structurel acteurenvironnement. Il s'agit de la familiarité de l'acteur à lui-même, et sa présence à soi continue accompagnant le flux de son activité (Durand, 2008, p. 99). La caractéristique de vécu de l'activité permet à tout acteur, moyennant des conditions favorables, de restituer ce qui fait expérience à chaque instant en montrant, racontant, commentant, simulant, mimant son activité (Theureau, 2006). Grâce à ce postula, il devient possible d'accéder à la cohérence et l'intelligibilité de l'activité de chaque observatrice, à partir leur du point de vue.

\section{Hypothèse 3 : l'activité signe}

L'activité signe traduit la pensée-signe (Pierce, 1931) en termes d'activité humaine (Theureau, 2006). Ce pré-supposé considère le flux de l'activité humaine comme une concaténation, un enchâssement de signes pris en compte et produits par l'acteur. Le repérage de l'enchainement des signes rend compte de la construction des significations pour l'acteur, nommée cours d'expérience. Il permet une description de l'activité humaine et une description de comment cette activité donne lieu à expérience (Theureau, 2015). Ce postulat sémiologique nous fournit les méthodes d'analyses des données recueillies sur la base des composantes du signe, repris et enrichi de la notion de signe triadique de Pierce (Pierce, 1931). Pour notre étude, nous avons retenu une description du contenu des signes et de leur enchainement, à partir des six pôles fondamentaux constituant le signe hexadique: l'engagement(E), l'actualité 
potentielle (A), le référentiel (S), le representamen (R), l'unité élémentaire de cours d'expérience (U) et l'interprétant (I). Nous définissions chaque composante afin de rendre plus explicite notre étude (Theureau, 2015) :

- l'engagement (E) de l'acteur dans la situation. Il traduit le faisceau de préoccupations, de présuppositions qui s'ouvrent à l'instant $t$ pour l'acteur et qui sont en relation avec son activité antérieure.

- l'actualité potentielle (A). Elle traduit la délimitation des attentes potentielles de l'acteur dans la situation à l'instant $t$, soit d'une préparation de son futur pour l'acteur.

- le référentiel (s). C'est l'ensemble des savoirs (nommés types) non figés et hérités de l'histoire des couplages vécus, qui sont mobilisables par l'acteur à l'instant $t$, compte tenu des deux composantes $\mathrm{E}$ et $\mathrm{A}$.

Les composantes E, A, S rendent compte de l'histoire des interactions passées et futures, pour la compréhension de la dynamique de construction de l'expérience.

- le représentamen (R) est ce qui «fait signe», ce qui est perturbateur pour l'acteur à l'instant $t$, dans sa situation, compte tenu de la structure d'anticipation $\mathrm{A}$.

- L'unité élémentaire du cours d'expérience (U) traduit l'émergence pour l'acteur d'une conscience pré-reflexive accompagnant l'activité qui découle de la perturbation R. Cette unité peut être une action, une communication, une focalisation, une interprétation, une imagination ou une émotion.

Les composantes $\mathrm{R}$ et $\mathrm{U}$ rendent compte de la dynamique de la situation de l'acteur, pour la compréhension de la dynamique de construction de l'expérience.

- L'interprétant (I) traduit l'hypothèse de la constante transformation, à divers degrés du savoir de l'acteur et de ses habitudes situées accompagnant son activité. Il est une élaboration continue de construction, validation ou invalidation de types et relation entre types par l'acteur en situation.

La composante I rend compte de la dynamique des apprentissagesdéveloppements, pour la compréhension de la dynamique de construction de l'expérience.

En adoptant ces présupposés, l'étude mise en place a pour objectif de produire des connaissances scientifiques sur l'activité des observatrices en considérant sa totalité dynamique (composée des 6 pôles) et en respectant le principe du primat de l'intrinsèque. L'activité d'observation est appréhendée dans une perspective dynamique, non stabilisée dont la transformation émane des activités typiques en amont et en aval de celle-ci. Le postulat étant que cette activité possède une organisation sous-jacente dont il est possible de dégager des régularités et/ou des singularités entre les différentes expériences de visionnage.

\subsection{Méthodologie}

\subsubsection{Contextualisation de l'étude}

L'étude se déroule sur le plateau technique du SIMUH-Nîmes dédié à la simulation en obstétrique et néonatologie. Elle s'intéresse à l'activité déployée par des étudiantes en maïeutiques, observatrices ${ }^{7}$ de leurs paires, lors d'une Unité d'Enseignement nommée "Simulation: urgences obstétrico-pédiatriques». Les objectifs généraux de la formation sont :

- de placer les étudiantes en situation de responsabilité ;

- d'expérimenter, appréhender la complexité d'une situation d'urgence; 
- d'être capable d'appliquer, en situation critique les procédures et manœuvres préconisées selon les recommandations actuelles;

- de développer des compétences sociales;

- de développer une culture d'analyse des pratiques.

Ce module de cinq séances, réparties sur un semestre, s'adresse à un groupe de 5 étudiantes sages-femmes, de quatrième année. Chaque étudiante se porte volontaire pour être actrice de deux situations simulées, une fois en qualité de sage-femme et une fois en qualité d'aide-soignante. Elles sont observatrices lors des trois autres séances. Les scénarios prévus par les formateurs ne sont pas connus des participantes, afin que le diagnostic de la situation problème soit réalisé pendant la pratique clinique, comme c'est le cas en situation réelle. La conduite des séances respecte le découpage type décrit précédemment. Les observations des situations simulées sont médiées par une transmission audio-vidéo en direct en salle de débriefing, sans présence de formateurs. $\mathrm{Au}$ total, 13 situations d'observation ont été analysées.

\subsubsection{Observatoire}

\section{Recueil des données}

37 Selon le cadre théorique cours d'action, les données observables du couplage observatrices-environnement, doivent être croisées à la description abstraite de l'expérience à instant $t$ (Theureau, 2006). La construction des données s'est réalisée à deux niveaux.

Un niveau de description documentant l'activité manifeste des observatrices à partir de différentes traces : vidéo-audio-enregistrements (voir photo 1) de la situation observée (voir photo 2) et de la situation d'observation (voir photo 3) et photographies des productions écrites (voir photo 4). Ces supports visent d'une part à resituer les observatrices dans la situation étudiée, lors des entretiens d'autoconfrontation réalisés en décalage temporel et d'autre part à constituer des données comportementales et contextuelles indispensables à l'analyse des cours d'actions. 
Photo 1 : Salle de débriefing et son équipement pour le recueil des données. Photo 1 : Debriefing room and its data collection equipment

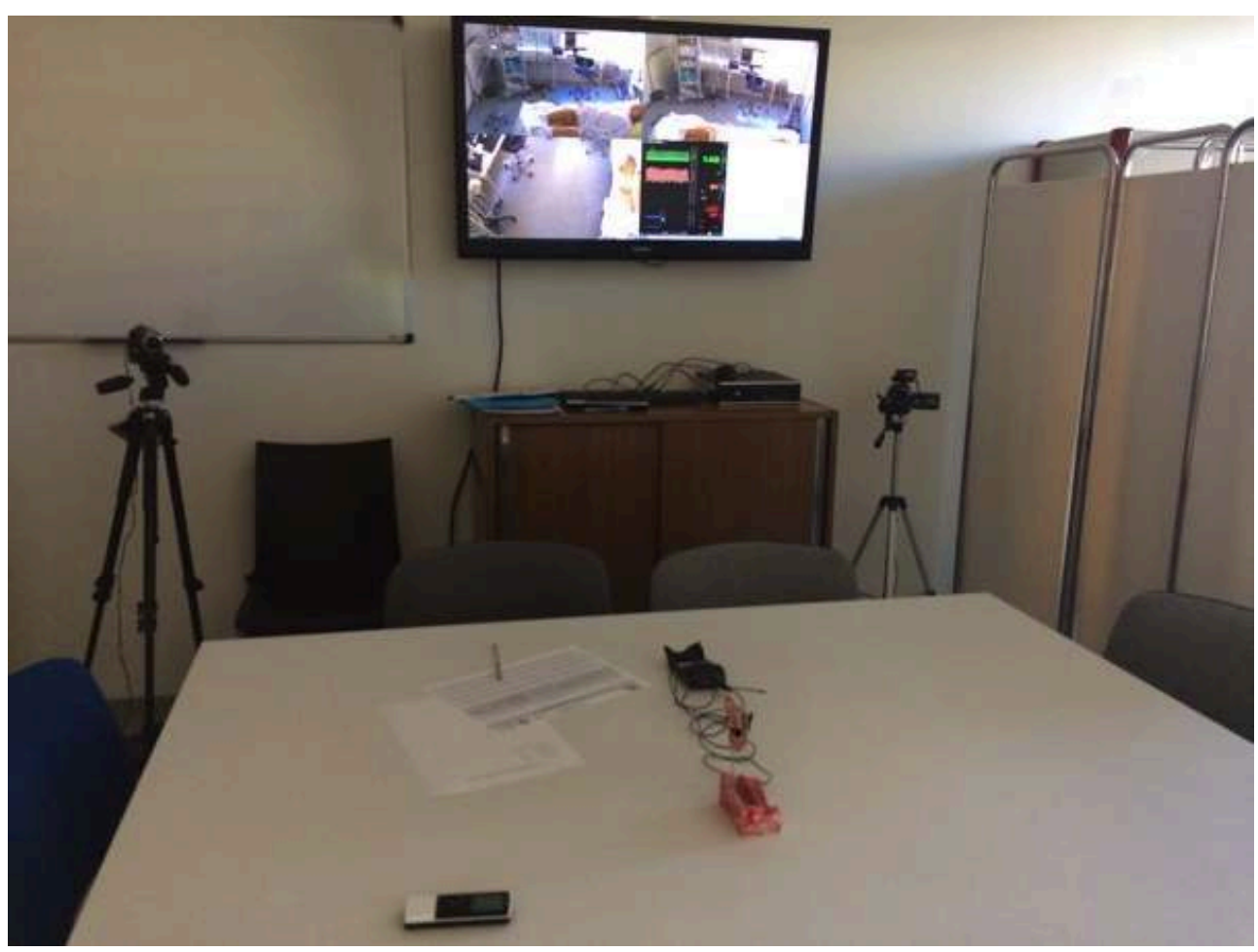

Photo 2 : Écran de projection à quatre entrées, observé lors d'une séance de SHF. Photo 2 : Four-screen projection observed during a High Fidelity Simulation (HFS)

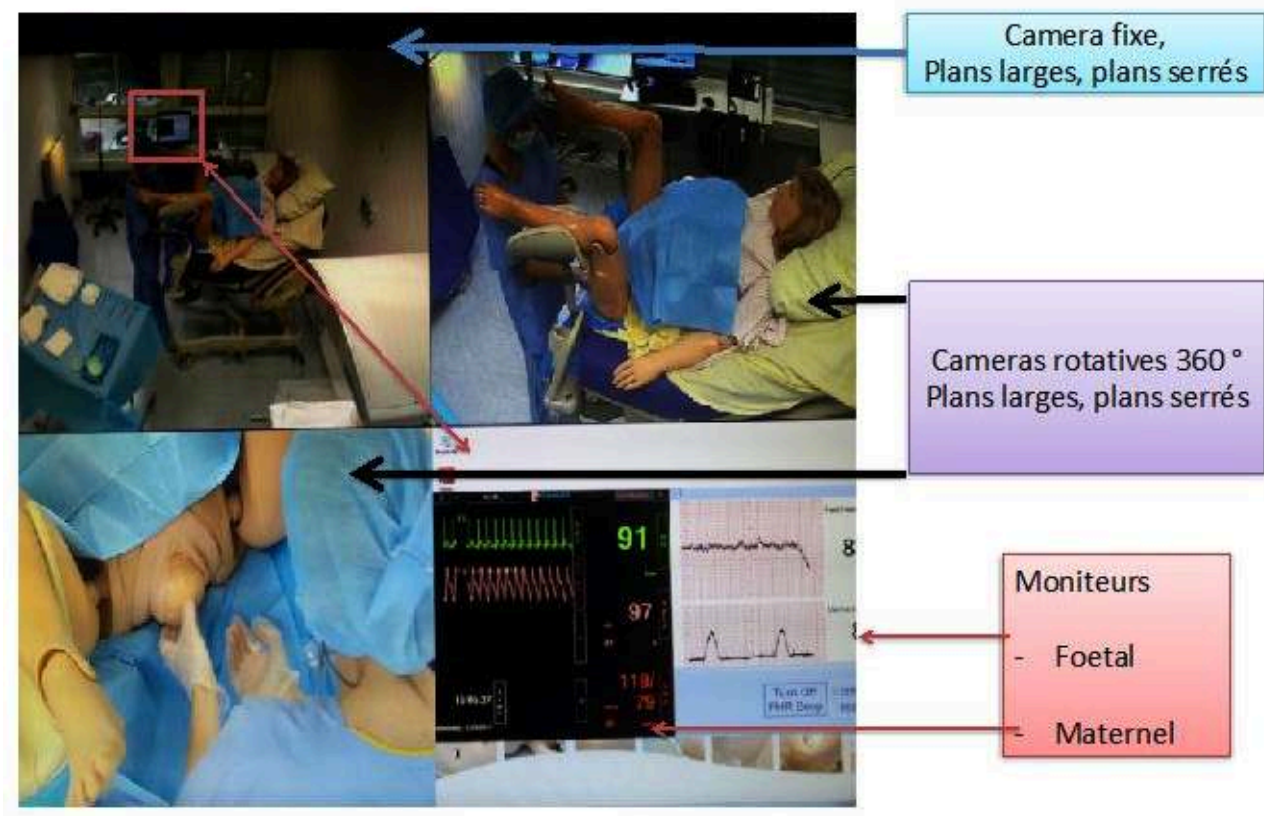


Photo 3 : Enregistrement audio-vidéo de trois observatrices.

Photo 3 : Audio/video recording of three observers

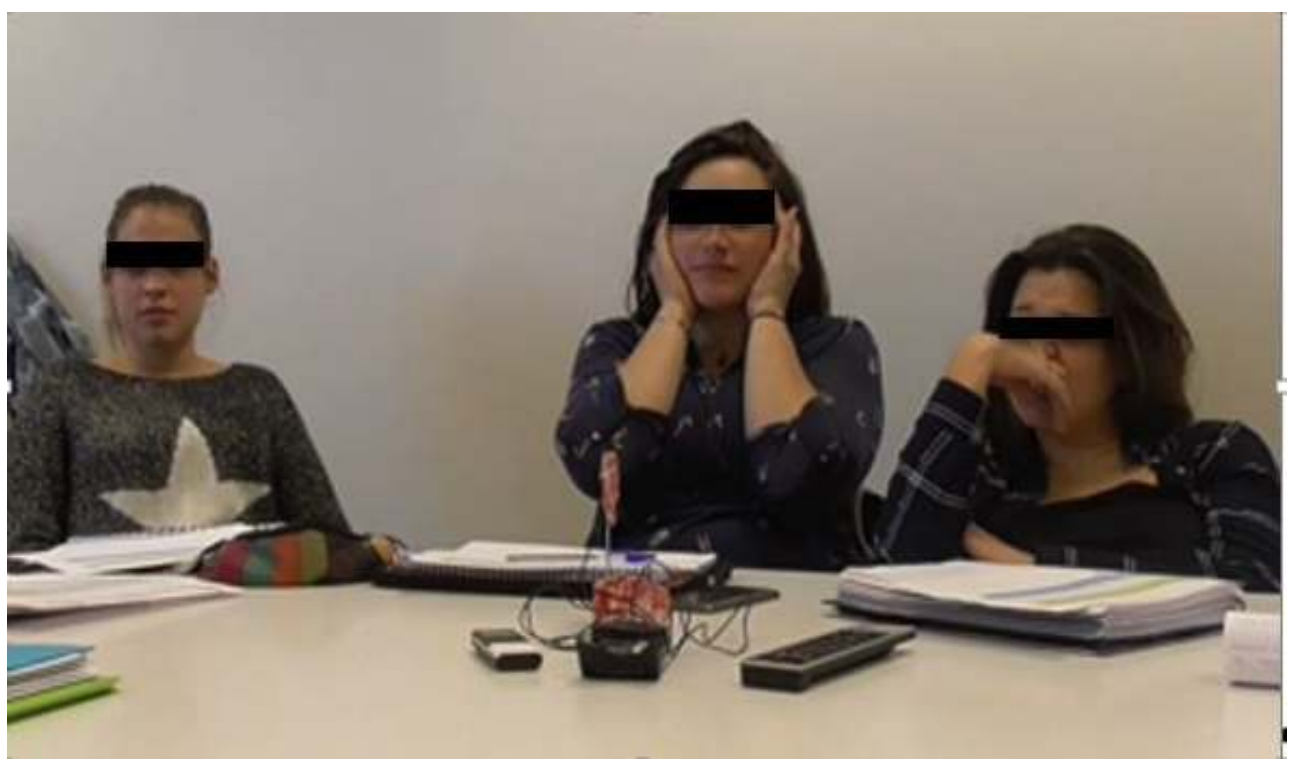

Photo 4 : Traces de l'activité d'écriture.

Photo 4 : Traces of the writing activity

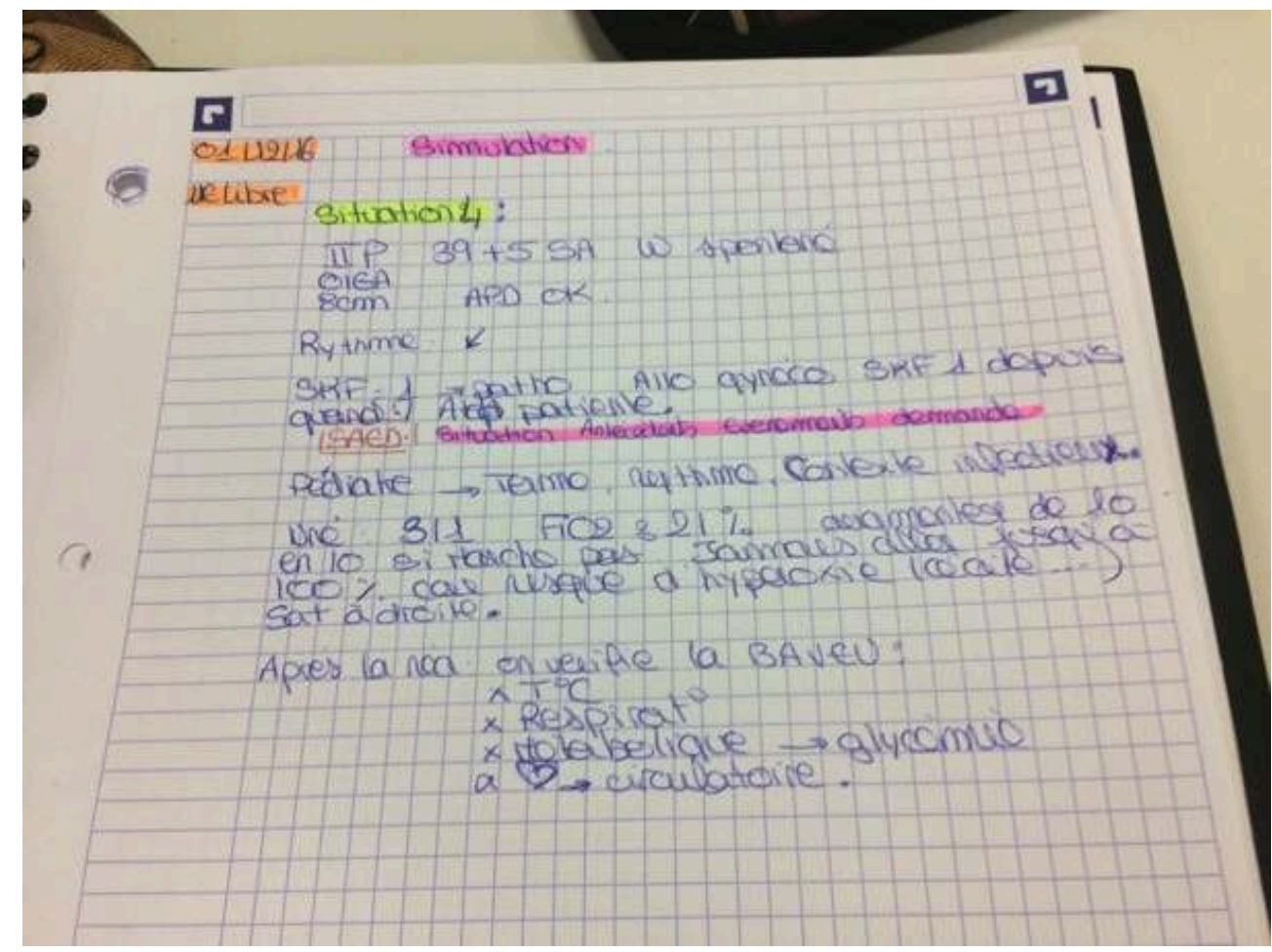

Un niveau de description documentant la partie invisible de l'activité à travers la verbalisation du vécu des observatrices lors des entretiens d'auto-confrontations simples, interruptifs, filmés (voir photo 5), réalisés dans la semaine suivant la séance de simulation. Les données filmiques de la situation d'observation et de la situation de simulation ont été synchronisées. Ces données avec les traces écrites participent à une remise en situation dynamique des observatrices «ici et maintenant». Cette 
instanciation leur permet de documenter les aspects implicites, opaques, silencieux de l'activité et sa construction située.

Photo 5 : Entretien d'autoconfrontation audio-vidéoscopé.

Photo 5 : Audio-videoscopic self-confrontation interview

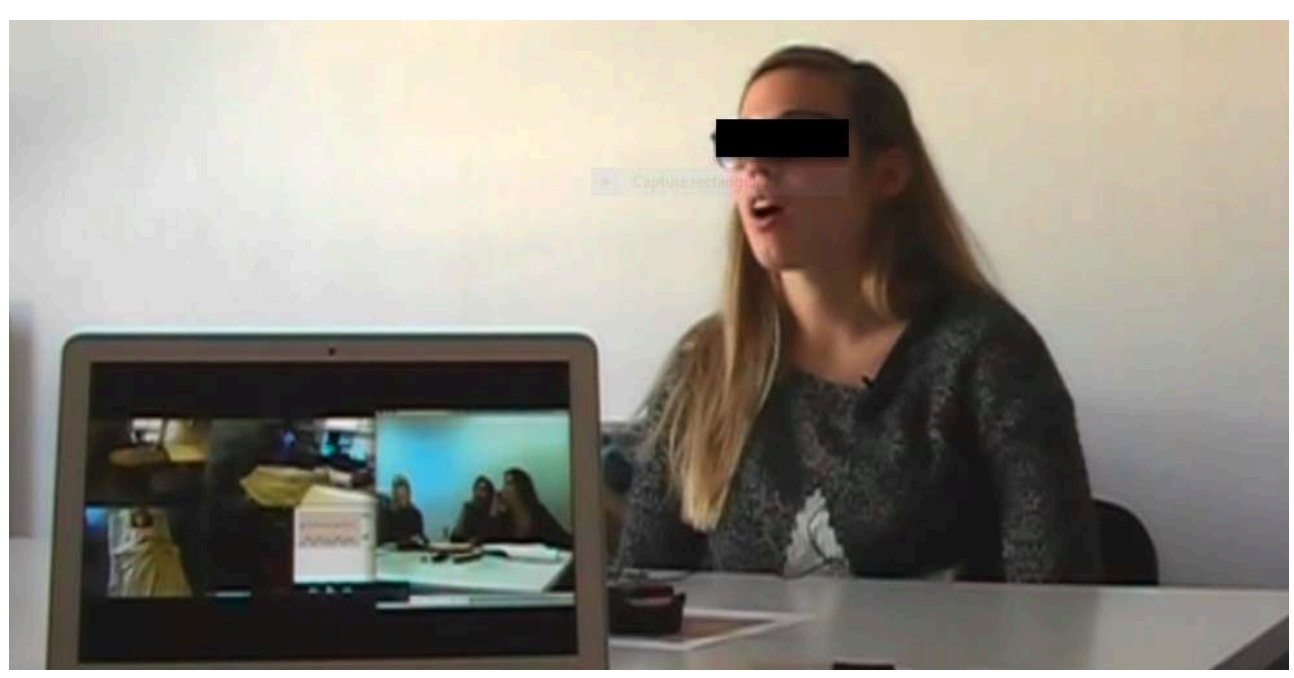

Traitement des données : construction d'un protocole à trois volets

L'ensemble des données filmiques ont été transcrites sous forme d'un protocole à trois volets (voir Tableau 1) mettant en correspondance les verbatim de la situation de simulation (volet ou colonne 1), celles de le la situation d'observation (volet 2) et celles des auto-confrontations (volet 3). Les traces écrites et des illustrations photographiques complètent le volet 2 . 
Tableau 1 : Extrait d'un protocole à trois volets. Chart 1 : Extract from a three-part protocol

\begin{tabular}{|c|c|c|}
\hline Volet 1 & Volet 2 & Volet 3 \\
\hline $\begin{array}{l}\text { Situation de simulation } \\
\mathbf{n}^{\circ} 5^{8} \\
\text { Durée : } 19 \mathrm{mn} 10 \\
\text { Acteurs : } \\
\text { C: }: \text { age-femme } \\
\text { E: Aide-soignante (AS) } \\
\mathrm{N}: \text { Noëlle : patiente : mannequin } \\
\text { haute-fidelité } \\
\text { HB : formateur, facilitateur }\end{array}$ & $\begin{array}{l}\text { Situation d'observation } \\
\text { Durée : } 19 \mathrm{mn} 43 \\
\text { Observateurs } \\
\text { A, L M : étudiantes observatrices } \\
\text { /= action synchrone } \\
\text { ๔ = regarde la projection vidéo }\end{array}$ & $\begin{array}{l}\text { Entretien d'auto-confrontation } \\
\text { Durée: } 01 \mathrm{~h} 17 \mathrm{mn} 13 \\
\text { HB : chercheur } \\
\text { A : etudiante réalisant l'entretien } \\
\text { d' autoconfrontée } \\
\text { M= stop vidéo } \\
\mathbf{A}=\text { lancement vidéo }\end{array}$ \\
\hline $\begin{array}{l}01.45 \text {. C: tu adaptes le synto } \\
\text { pour la délivrance dirigée } \\
\mathrm{E}: \text { on va adapter ça... (connecte } \\
\text { la seringue à la ligne de } \\
\text { perfusion)... C'est bien si vous } \\
\text { êtes reposée, vous allez être en } \\
\text { forme pour pouvoir pousser... } \\
\mathrm{N} \text { : ah oui c'est sur } \\
\mathrm{E}: \text { vous avez quatre grands } \\
\text { enfants c'est ça? } \\
\mathrm{N}: \text { oui...j'ai trois filles et un }\end{array}$ & 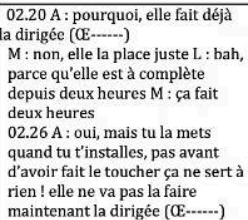 & $11.32=\mathrm{A}$ : donc là, f'a \\
\hline $\begin{array}{l}\text { garçon } \\
\text { E: } 3 \text { filles } \\
\text { quel âge? }\end{array}$ & & 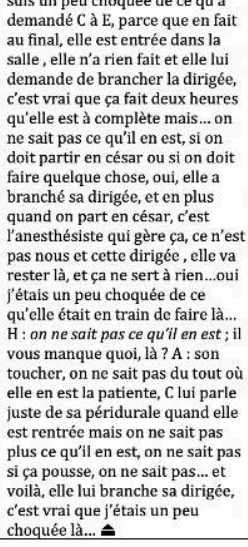 \\
\hline
\end{tabular}

\section{Étapes analytiques}

41 La méthode cours d'action comporte trois dimensions complémentaires : le découpage -étiquetage des unités (dimension synthétique), la catégorisation des unités (dimension analytique) et l'identification d'invariants, de variations et de facteurs de variation (dimension comparative) (Theureau, 2006).

- Analyse locale : étiquetage des unités significatives et de leurs composantes (E-A-S-R-U-I)

La discrétisation des cours d'expérience permet de restituer la dynamique de concaténation des signes pris en compte et produits par les observatrices. Cette démarche s'appuie sur l'activité des observatrices (volet 2), renseignée du point de vue de chaque observatrice (volet 3), c'est à dire de ce qui est significatif pour chacune dans la situation observée (volet 1$)$. 
Tableau 2 : Illustration de la documentation d'un signe à partir du tableau 1. Chart 2 : Illustration of sign documentation from chart 1

\begin{tabular}{|c|c|c|c|c|c|}
\hline $\mathrm{E}$ & A & $S$ & $\mathrm{R}$ & U14 02'20" & I \\
\hline $\begin{array}{l}\text { Rester attentive à } \\
\text { ce que font et } \\
\text { disent } C \text { et } E \\
\text { Évaluer les } \\
\text { actions de C }\end{array}$ & $\begin{array}{l}\text { A s'attendant à ce } \\
\text { que } C \text { fasse un } \\
\mathrm{TV}^{9} \text { à la patiente } \\
\begin{array}{l}\text { A anticipant un } \\
\text { départ en } \\
\text { césarienne }\end{array}\end{array}$ & $\begin{array}{l}\text { [Brancher la } \\
\text { délivrance } \\
\text { dirigée] implique } \\
\text { d'être sûre que } \\
\text { l'accouchement } \\
\text { va se faire par } \\
\text { voie basse } \\
\text { [En cas de } \\
\text { césarienne, la } \\
\text { délivrance } \\
\text { dirigée] est gérée } \\
\text { par l'anesthésiste } \\
\text { [Faire un TV] } \\
\text { permet de savoir } \\
\text { "où en est la } \\
\text { patiente" }\end{array}$ & $\begin{array}{l}\text { C dit « tu adaptes } \\
\text { le synto pour la } \\
\text { délivrance } \\
\text { dirigée (DD) » }\end{array}$ & $\begin{array}{l}\text { Pose une } \\
\text { question en } \\
\text { regardant l'écran } \\
\text { Communication: } \\
\text { demande } \\
\text { pourquoi C "fait } \\
\text { déjà la dirigée" } \\
\text { Discours } \\
\text { intérieur: se dit } \\
\text { que C devrait } \\
\text { faire un TV avant } \\
\text { de s'occuper de la } \\
\text { DD } \\
\text { Émergence de } \\
\text { sentiment: est } \\
\text { choquée de ce } \\
\text { que fait C }\end{array}$ & $\begin{array}{l}\text { Émergence du } \\
\text { type: [brancher la } \\
\text { DD sans avoir fait } \\
\text { de TV] est } \\
\text { inadapté }\end{array}$ \\
\hline $\begin{array}{c}\mathrm{E} \\
\text { Évaluer ensemble } \\
\text { les décisions de C }\end{array}$ & \begin{tabular}{l}
\multicolumn{1}{c}{ A } \\
A s'attendant à ce \\
que C fasse un TV \\
à la patiente
\end{tabular} & $\begin{array}{l}\text { S } \\
\text { [Brancher la } \\
\text { délivrance } \\
\text { dirigée] implique } \\
\text { d'être sûre que } \\
\text { l'accouchement } \\
\text { va se faire par } \\
\text { voie basse }\end{array}$ & $\begin{array}{l}\text { R } \\
M \text { et } L \text { "ça fait } \\
\text { deux heures" }\end{array}$ & \begin{tabular}{l}
\multicolumn{1}{c}{ U15 02'26" } \\
Échange avec M \\
et L en regardant \\
l'écran \\
Communication : \\
argumente qu'il \\
faut faire un TV \\
avant de \\
brancher la DD \\
Émergence de \\
sentiment: est \\
choquée de ce \\
que fait C
\end{tabular} & \begin{tabular}{l}
\multicolumn{1}{c}{ I } \\
Émergence / \\
renforcement du \\
type: [brancher la \\
DD sans avoir fait \\
de TV] est \\
inadapté
\end{tabular} \\
\hline
\end{tabular}

- Analyse globale : dimensions analytiques et comparatives comprendre la cohérence d'ensemble de l'organisation de l'activité, cette étape permet de réduire la complexité de l'activité en unités significatives de rangs supérieurs aux unités du récit réduit (voir Tableau 2). La méthode consiste à identifier et modéliser, pour chaque étudiante, lors de chaque situation d'observation, les différents engagements (E) qui accompagnent le visionnage (Theureau, 2004).

Nous avons procédé en l'identification de :

- Séquences lorsqu'entre au moins deux signes, il y a ancrage du representamen dans le ou un signe précédent (relation de cohérence séquentielle) et que les préoccupations de ces signes sont de même nature (illustration voir annexe 1);

- Macro-séquences lorsqu'il existe une relation de compositions entre des séquences c'est à dire qui partage un niveau de préoccupation supérieur (voir colonne 2 de la modélisation, annexe 2);

- Séries lorsque des séquences et/ou macro-séquences partagent un niveau de préoccupation plus globale, mais sans entretenir de relation de cohérence séquentielle (voir colonne 1 de la modélisation, annexe 2);

La présentation synthétique des modélisations permet de visualiser les continuités et discontinuités dans le flux de l'activité globale. Elle facilite l'identification d'éventuels temps de rupture orientant une analyse itérative entre les données modélisées, les données locales, les traces de l'activité enregistrées et leurs transcriptions sous forme de protocoles à trois volets. L'ensemble des cours d'actions a été comparé afin d'identifier des similarités et comprendre comment l'activité des observatrices s'organise dans cet espace du dispositif de formation. L'ensemble des séquences et 
macro-séquences identifiées a été comptabilisé et traduit en pourcentage. Ces données quantitatives, associées à l'analyse qualitative, facilitent l'identification de spécificités ou de généralités entre les descriptions de l'organisation intrinsèque de l'activité des étudiantes.

\section{Résultats}

L'analyse des cours d'expérience (CE) a permis d'identifier des structures significatives de différents rangs occurrentes à l'ensemble des modélisations. Elles présentent toutefois certaines variations selon les situations observées et les étudiantes. Il a été dégagé que l'activité des observatrices s'organise de façon typique en deux phases : une phase exploratoire avec un engagement co-déterminé par la recherche du diagnostic de la situation problème puis une phase exécutoire visant la résolution du problème posé par la situation. Les résultats présentent les engagements typiques aux étudiantes avant puis après le diagnostic confirmé de la situation problème. Dans un troisième temps sont documentées les préoccupations typiques qui accompagnent l'activité d'observation tout au long du visionnage, c'est à dire qui émergent indifféremment avant et après la découverte du scénario prévu par les formateurs. Les données locales affinent les données globales et permettent d'identifier :

- des facteurs de variations de l'activité;

- des phénomènes participant à des variations du jeu de proximité distance avec la situation observée, propices à l'activité réflexive;

- des processus de transformations liées à l'activité de référence de la sage-femme et au dispositif de formation.

\subsection{Un engagement dans l'activité différencié par l'indétermination puis la détermination de la situation simulée}

47 La discrétisation des cours d'expérience a permis de repérer un temps de rupture dans l'activité des observatrices. La découverte de la situation problème, prévue par les formateurs, s'accompagne d'une transformation des engagements typiques initiaux, principalement de nature exploratoire et engagements typiques de nature exécutoire. Ces transformations sont illustrées, en annexes 2 et 3, par les analyses globales de l'activité d'observation d'une étudiante, avant et après diagnostic de la pathologie (scénario d'hémorragie de la délivrance).

\subsubsection{Un engagement typique dans l'activité porté par l'intrigue du scénario}

L'activité d'observation, avant le diagnostic de la situation problème, est caractérisée par une préoccupation de découverte occurrente à l'ensemble des observatrices et des situations. Elle est identifiée par la série "découvrir collaborativement la situation problème prévue par les formateurs». Cette série est composée de trois macroséquences, trois préoccupations qui organisent l'activité de découverte :

- la macroséquence «Diagnostiquer la situation problème ». Elle traduit l'anticipation dans la situation observée, d'un évènement incidentiel prévu mais indéterminé. Le référentiel (S) de chaque observatrice comporte un principe commun [situation de 
simulation] : est une situation problème, cohérente avec la dénomination d'« urgences obstétriopédiatriques » de l'unité d'enseignement. L'indétermination de la situation est source de perturbation. La préoccupation de diagnostic s'accompagne, pour toutes les étudiantes, de projections hypothétiques de scénarios possibles avec des variations selon les situations et les étudiantes. Par exemple pour la situation d'hémorragie de la délivrance, cette macroséquence émerge dans $32 \%$ des signes pour l'étudiante A (cf. annexe 2) et dans 50 et $60 \%$ des signes pour les deux autres étudiantes. Cette variation pour l'étudiante A, s'explique par le fait qu'elle ne relève aucun élément prédictif d'une pathologie dans la situation observée. La parturition ${ }^{8}$ et l'accouchement sont physiologiques. Il ressort que l'activité d'enquête de la situation problème ne peut s'ancrer dans des données cliniques signifiantes pour elle. Il s'en dégage une phase de latence, d'expectative s'accompagnant de l'émergence du type [situation de simulation physiologique]: génère de la déception, de la frustration car ce "n'est pas le plus formateur » et d'une remise en cause du principe [situation de simulation]: est une situation problème.

- La macroséquence «Rester attentive, se concentrer». Elle traduit l'immersion. Elle émerge dès le lancement de la projection et est permanente. Elle est documentée dans plus de $80 \%$ des signes, pour l'ensemble des modélisations. Les séquences qui la constituent montrent des focalisations spécifiques aux situations pour recueillir des indices. Par exemple dans la situation d'arrêt cardio-respiratoire maternelle, la séquence «rester attentive à la patiente » est prépondérante (environ $50 \%$ des signes), alors qu'elle est quasi absente dans la situation de réanimation du nouveau-né, pour laquelle la séquence « rester attentive au monitoring fotal » émerge dans $53 \%$ à $65 \%$ des signes.

Tableau 3 : Illustrations photo et documentations du phénomène d'immersion en situation. Chart 3 : Photo illustrations and documentations of immersion phenomenon

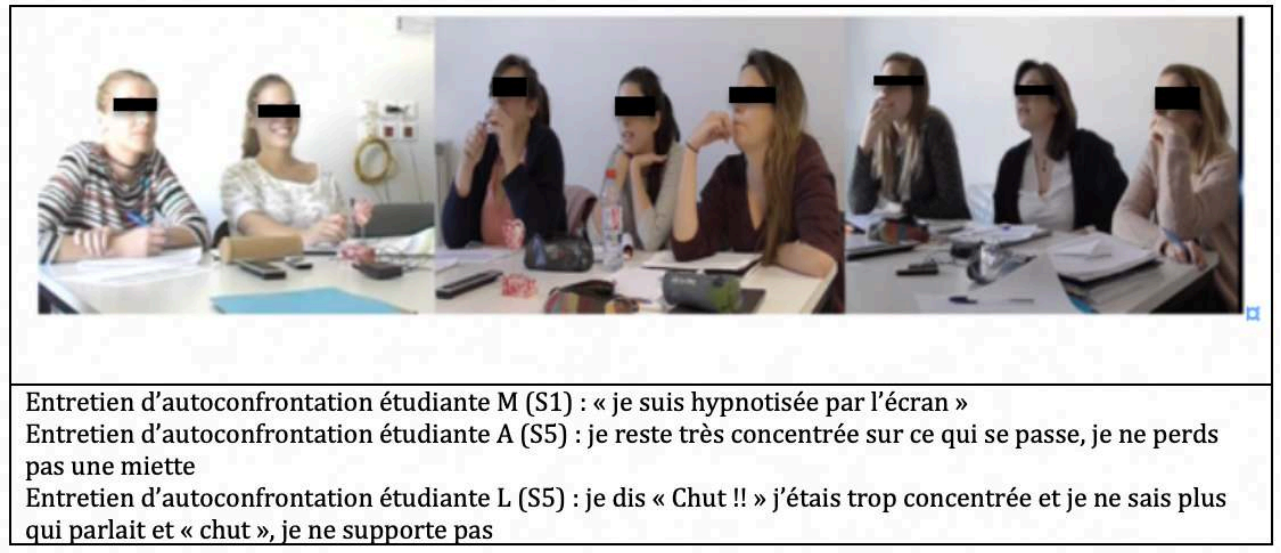

- La macroséquence "partager ce qui émerge de son observation ". Elle traduit l'engagement coopératif ' des étudiantes dans l'activité d'enquête. Ainsi, l'observation, qui n'est pas solitaire, est saisie comme l'opportunité de travailler de concert et de s'entraider pour atteindre un même objectif : découvrir la nature de la situation problème. Elle est documentée, pour chaque modélisation, dans plus de $40 \%$ des signes. L'activité coopérative se manifeste par la mutualisation des recueils d'indices et de constructions d'hypothèses diagnostiques, identifiée dans la composante U par les communications-types étiquetées «verbalise son constat", "verbalise son hypothèse", «apporte une réponse au questionnement d'une observatrice », « confirme ou infirme ce que dit une observatrice » ou encore « partage son questionnement». 
Les engagements typiques aux observatrices, révélés par ces macro-séquences, s'actualisent en une activité de raisonnement clinique prenant en compte des données propres à la situation clinique mais également des éléments spécifiques à la situation simulée ou à la formation par simulation. En effet, les présupposés techniques du mannequin et l'anticipation de certains scénarios jugés emblématiques, documentés dans le référentiel $(\mathrm{R})$, orientent l'analyse.

(S5-obsA) : S de U49 : [scénarios proposés par les formateurs] : ne peuvent pas être deux fois les mêmes.

(S1-ObsL) : $\mathrm{S}$ de U2 : [possibilités techniques du simulateur] : ne permettent pas de simuler une hémorragie de la délivrance.

Il en découle des pronostics de situations consistant en une logique probabiliste de situations problèmes produite par ces principes et par les faits retenus comme pertinents en situation. Les indices sélectionnés par les étudiantes sont interprétés selon trois processus du raisonnement clinique :

- le processus analytique conditionnel par lequel l'observatrice repère un ensemble de données dont le chainage met en évidence un lien de familiarité avec des expériences antérieures, aboutissant au diagnostic

Autoconfrontation : Étudiante C-S3 : elle a dit «j'ai mal à la poitrine et dans le bras », et là c'est au même moment ou j'ai vu les pulsations, zéro, zéro, elle est en arrêt.

- le processus analytique hypothético-déductif par lequel l'observatrice pose une hypothèse et recherche des indices pertinents pour l'infirmer

Autoconfrontation : Étudiante $\mathrm{M}-\mathrm{S} 5$ : là, je reviens à l'hémorragie, je me dis, ça ne s'arrête pas à un accouchement simple, je ne voyais pas d'autre situation possible.

- le processus non analytique : l'observatrice identifie immédiatement la similitude de la situation avec une expérience antérieure

Autoconfrontation : Étudiante $\mathrm{A}-\mathrm{S} 4$ : là, on découvre la situation on sait très bien qu'il va y avoir un problème sur le bébé.

51 L'engagement de découverte s'accompagne d'une forte activité interprétative indissociable du raisonnement clinique. Elle se caractérise, selon les observatrices, de mobilisations, renforcements et créations de connaissances en physiopathologie obstétricale ou pédiatrique et de connaissances procédurales, documentés par les interprétants (I).

(S4-obsE). I de U14 : Émergence du type : [appel au pédiatre] : implique de savoir interpréter le rythme mais aussi de savoir " comment décrire le rythme ».

(S4-obsA). I de U3 : Gain de validité du type : [absence de variabilité] : ne peut pas être assimilée à une phase de sommeil, «je n'ai jamais vu un rythme comme ça quand bébé dormait ».

52 L'activité est spécifiée par un engagement compréhensif de la situation visionnée, avec expériences interprétatives partagées, que nous qualifions d'engagement exploratoire. Elle s'accompagne de processus de transformations relatifs à la pratique de la maïeutique et au dispositif de formation. L'engagement exploratoire s'achève lorsque la situation est déterminée. La macro-séquence "découvrir la situation problème " disparaît des modélisations. 


\subsubsection{Un engagement typique dans l'activité porté par le dénouement du scénario}

Le diagnostic confirmé de la situation prévue par les formateurs s'accompagne d'une nouvelle préoccupation. La série "résoudre la problématique de la situation » est occurrente à l'ensemble des modélisations. Elle est composée de plusieurs macroséquences :

- La macro-séquence « rester attentive ". Elle est présente dans plus de $90 \%$ des signes des modélisations post diagnostic et traduit comme pour la phase exploratoire l'implication quasi permanente des étudiantes dans l'activité d'observation, leur immersion dans la situation observée.

- La macro-séquence «vivre la situation » est présente dans tous les cours d'expérience post diagnostic, mais présente des variations selon les étudiantes et les situations observées. Elle traduit l'expérience de nature efficiente des étudiantes qui élaborent des choix d'actions et s'engagent corporellement en situation observée. Elle est constituée des séquences « définir ce qu'il faut faire » et « se placer en situation et s'imaginer agir » qui s'enchâssent ou se synchronisent selon les situations. La séquence "définir ce qu'il faut faire " est présente dans plus de $50 \%$ des modélisations post diagnostic. Elle s'actualise en une activité de prises de décisions d'actions jugées pertinentes pour corriger la dégradation du patient. Ces décisions sont documentées dans les unités de cours d'expérience (U).

(S4-obsE). U80 : Communication : là il faut faire un massage cardiaque.

(S4-obsL). U95 : Discours intérieur : se dit qu'avec une saturation à $83 \%$; c'est bon, il n'y a plus rien à faire à par ventiler.

La séquence «se placer en situation et s'imaginer agir " s'actualise en expérience par procuration. Les observatrices s'imaginent à la place de leurs collègues et se projettent agir. Ces expériences émergent de l'observation lorsque la situation est jugée critique, que les actions observées soient inadéquates ou qu'il y a nécessité de réaliser des manœuvres complexes pour dégager le fotus ou stabiliser la patiente. L'expérience par procuration est documentée dans l'unité de cours d'expérience (U) et s'accompagne de deux phénomènes spécifiques aux épisodes jugés critiques :

- l'émergence d'émotions à valences négatives (ressent du stress, ressent de l'inquiétude, ressent de la peur) (illustrations voir Tableau 4). 
Tableau 4 : Illustrations photo de manifestations émotionnelles accompagnant la documentation de l'unité de cours d'expérience (U).

Chart 4 : Photo illustrations of emotional events accompanying the unit's documentation $(U)$

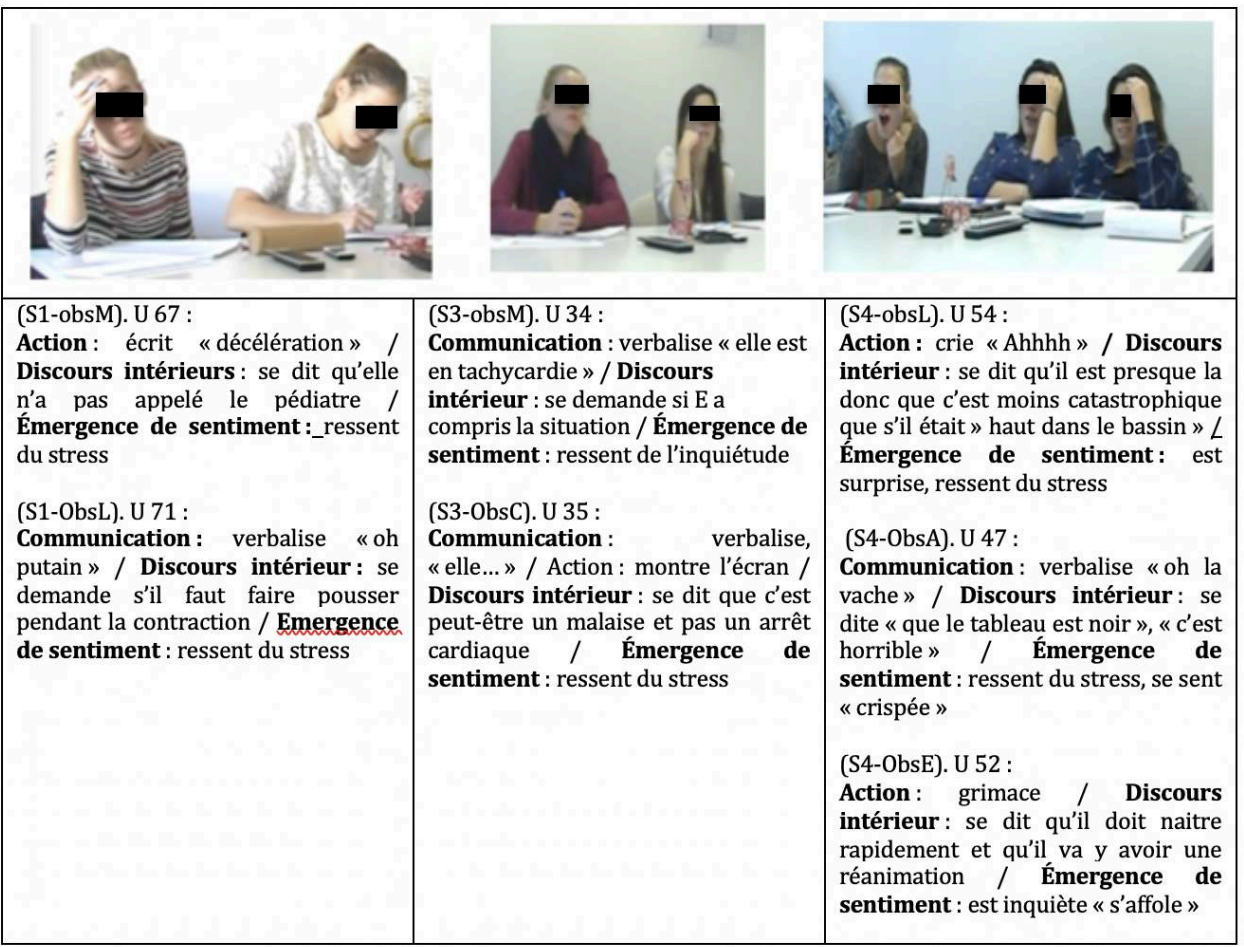

- la reproduction de manœuvres sans contact effectif avec le fœtus ou la patiente. Ces gestuelles accompagnent le langage, l'illustrent, le complètent. Ils sont adressés aux autres observatrices pour partager des connaissances et/ou auto-adressés pour s'entrainer à une pratique (illustrations voir Tableau 5). 
Tableau 5 : Chronophoto illustrant la reproduction de la manœuvre de retournement et accompagnant la documentation d'un signe (S1-ObsL).

Chart 5 : Chronophoto illustrating the reproduction of the reversal maneuver and accompanying the documentation of a sign

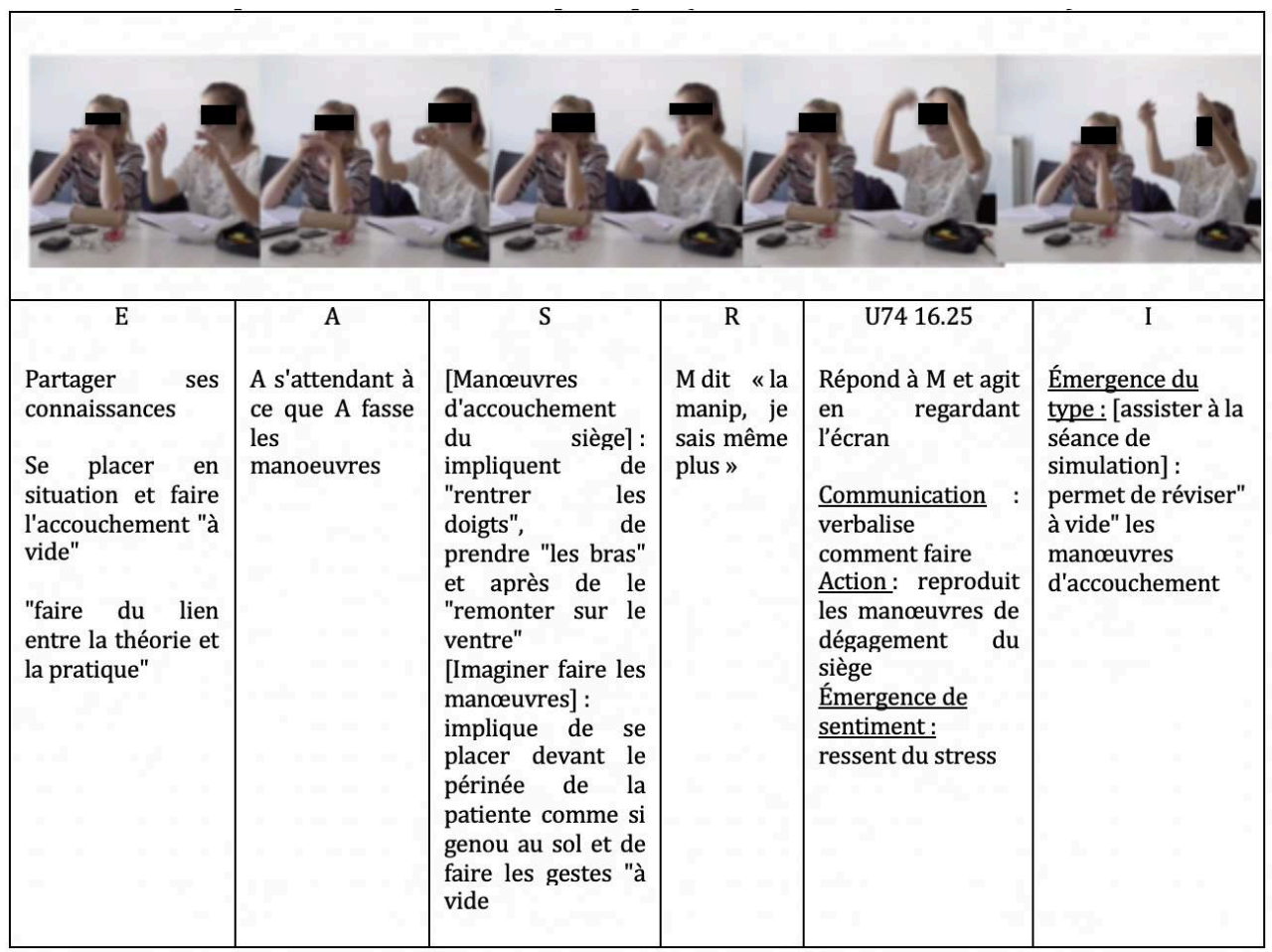

- La macro séquence « superviser ses paires" s'enchâsse ou se synchronise avec la séquence " définir ce qu'il faut faire ». Les étudiantes comparent ce qui serait fait ou ce qui est attendu, avec ce qui est vu faire (séquence «évaluer les actions de la sage-femme et l'aide-soignante » présente dans 40 à $57 \%$ des signes). Lorsque les actions observées sont en décalage avec celles jugées pertinentes, les étudiantes argumentent leurs choix décisionnaires ou les remédiations à faire en mobilisant leurs connaissances (référentiel S) (Illustration U15, Tableau 2). Il a été identifié que lors de phases critiques avec émergence d'émotion forte, les observatrices guident verbalement les actrices, leurs adressent des directives afin d'améliorer leurs performances ou éprouvent le besoin d'aller les aider (actualisation de la préoccupation de la séquence « aider la sage-femme »).

(S4-obsA). U 96 : Communication : «n'attends pas! Masse-le!».

(S3-obsC). U35 : communication : «non, non! Mets une plaque !».

(S1-Obsl). I de U65 : Émergence du type [être observateur] : implique d'avoir envie de lui souffler à l'oreille ce qu'il fait faire, donne trop envie d'aller le lui dire.

- Les macro-séquences «découvrir l'évolution de la situation " et "partager mutualiser » traduisent l'engagement récurrent d'évaluation de l'évolution de la situation permettant d'ajuster ou de confirmer les décisions prises.

Les situations d'urgences génèrent des émotions fortes associées à une contrainte temporelle pour agir. Les préoccupations des observatrices sont rattachées aux situations critiques présentes avec recherche de stabilisation. Par un jeu de proximité distance avec la situation et les actrices observées, les observatrices expérimentent des situations inédites et s'entrainent à engager des procédures et des actions pour améliorer l'état clinique des patients. L'activité s'accompagne de renforcements, 
transformations et de créations de connaissances procédurales en obstétricopédiatriques, de connaissances en mécanique obstétricales et d'habiletés techniques.

(S1-ObsM) : I de U81 : Gain de validité du type : [s'abstenir de «toucher » le fœetus, comme A] permet de ne pas devoir engager toutes les manœuvres.

(S2-ObsL) : I de U20 : Émergence du type : [scorer un rythme atypique] nécessite de le faire collégialement, pour s'assurer de la fiabilité de son analyse.

(S4-ObsE) : I de U 79: Émergence du type [ventiler avec le néopuff] ${ }^{10}$ nécessite de respecter le rythme " tu appuies 1 , avec l'index, et tu lâches 1-2 ».

Lors de la pratique en stage, les étudiantes ne gèrent jamais en toute autonomie les situations d'urgence. En simulation les étudiantes se retrouvent en première ligne, les observatrices découvrent la complexité de leur exercice futur.

(S1-ObsM). I de U68 : Émergence du type [devoir réaliser seule un accouchement du siège inopiné] : risque de mettre en danger la mère et le nouveau-né / Émergence du type [être observateur en simulation] : permet de «se projeter comme si c'était vrai »/ Émergence du type : [devoir gérer seule un accouchement du siège inopiné] : implique de devoir gérer le sentiment de peur, «d'avoir peur ».

(S4-ObsE) : I de U 14: Émergence du type [devoir appeler le pédiatre] me fait réaliser ne pas savoir quoi dire " comment décrire le rythme, de quelles info a-t-il besoin ».

Les étudiantes rapportent avoir vu un nombre limité d'hémorragie de la délivrance ou de réanimations du nouveau-né et ne jamais avoir rencontré les autres situations en stage. La macro-séquence "partager mutualiser» traduit l'instauration d'une coopération entre observatrices pour résoudre efficacement, ensemble, le ou les problèmes identifiés dans la situation. Cette préoccupation s'actualise par des communications-types, identifiées dans la composante U, étiquetées « verbalise ce qu'il faut faire", "verbalise ce qui serait fait", "confirme/infirme ce que dit une observatrice " ou encore "partage ses connaissances». Ces verbalisations s'accompagnent d'argumentions et/ou débats de choix décisionnaires, d'identifications de connaissances partagées et/ou de l'instauration de consensus de prise en charge. En effet, les connaissances construites en cours, ou lors des rares cas d'observation in situ, n'ont jamais pu être éprouvées. L'activité collégiale participe à leur accorder une plus forte validité ou à les transformer. Les interactions font émerger les instabilités ou les défauts de connaissances en mécanique obstétricale et en connaissances procédurales. Ces modifications constantes de la plausibilité des connaissances impliquent les observatrices dans un rapport à leurs propres connaissances documentées par certains interprétants (I).

(S2-ObsC) : I de U : Émergence du type [observer la séance de simulation] me permet de réaliser, que je ne connais pas toutes les manœuvres à réaliser en cas de dystocie des épaules, que " le Jacquemier ${ }^{11}$, je ne sais plus ce que c'est ».

(S4-ObsL) : I de U : Émergence du type [observer la séance de simulation] me permet de réaliser que « je ne me souviens plus » de la durée ventilation avant d'engager le massage.

Ces repérages incitent une implication dans les apprentissages à l'issue des séances de simulation (transformations projetées).

L'activité post diagnostic de situation est spécifiée par un engagement efficient relatif à la situation visionnée, que nous qualifions d'engagement exécutoire, avec expériences langagières et corporelles de natures descriptives, explicatives, argumentatives, interprétatives, exécutives. Elle se caractérise par des mises en relations entre l'activité observée et une activité autre, parallèle (activité de référence, activité vécue, activité 
propre imaginée) et s'accompagne de processus de transformations relatifs à l'activité professionnelle.

Nous avons décrit les engagements typiques qui organisent l'activité d'observation en deux phases et identifié certains facteurs de variations. Nous présentons maintenant un engagement typique des étudiantes participant, tout au long de la séance, à l'instauration de conditions propices à l'activité d'observation.

\subsubsection{Engagement typique dans l'activité traduisant l'instauration d'un lien social entre observatrices}

61 La série « créer des conditions propices à la conduite de l'activité » émerge tout au long de l'activité d'observation avec des variations selon les étudiantes et les situations observées. Elle se compose de :

- La macro-séquence « rester attentive à ce que font les observatrices ». Elle traduit, avec la macro-séquence "partager ses constats", la présence des étudiantes dans l'environnement d'observation qui est socialement constitué. Elle relève d'un engagement d'écoute et de soutien porté aux observatrices en présence, lors de questionnements, réflexions ou manifestions émotionnelles.

- La séquence "maintenir une connivence" est présente dans l'ensemble des cours d'expériences (7,5 à $13 \%$ des signes) et présente des variations selon les étudiantes et les situations observées. Elle est globalement plus présente lors de la phase exploratoire même si cette préoccupation ne s'éteint pas après le diagnostic de situation. Elle traduit la construction d'une complicité entre observatrices qui s'actualise par l'humour et le rire.

- La macro-séquence « s'amuser » accompagne tous les cours d'expérience. Comme pour la connivence, son émergence présente des variations, de 6 à $20 \%$ des signes, selon les situations et les étudiantes. Elle traduit, avec la séquence "maintenir une connivence », la disposition des étudiantes à instaurer une atmosphère cordiale propice à l'activité coopérative (enquête et résolution problème). Elle s'actualise par le rire, présent dans l'ensemble des cours d'expérience, et est documentée dans l'unité de cours d'expérience (U) (illustrations voir Tableau 6). Il émerge tantôt de l'activité de supervision, tantôt de la découverte de stratèges pour simuler certains évènements, ou encore des phénomènes de résonance ou d'expérience par procuration. 
Tableau 6 : Illustrations photo de manifestations de rires accompagnant la documentation de l'unité de cours d'expérience (U).

Chart 6 : Photo illustrations of laughter events accompanying the documentation of the course-ofexperience unit $(U)$

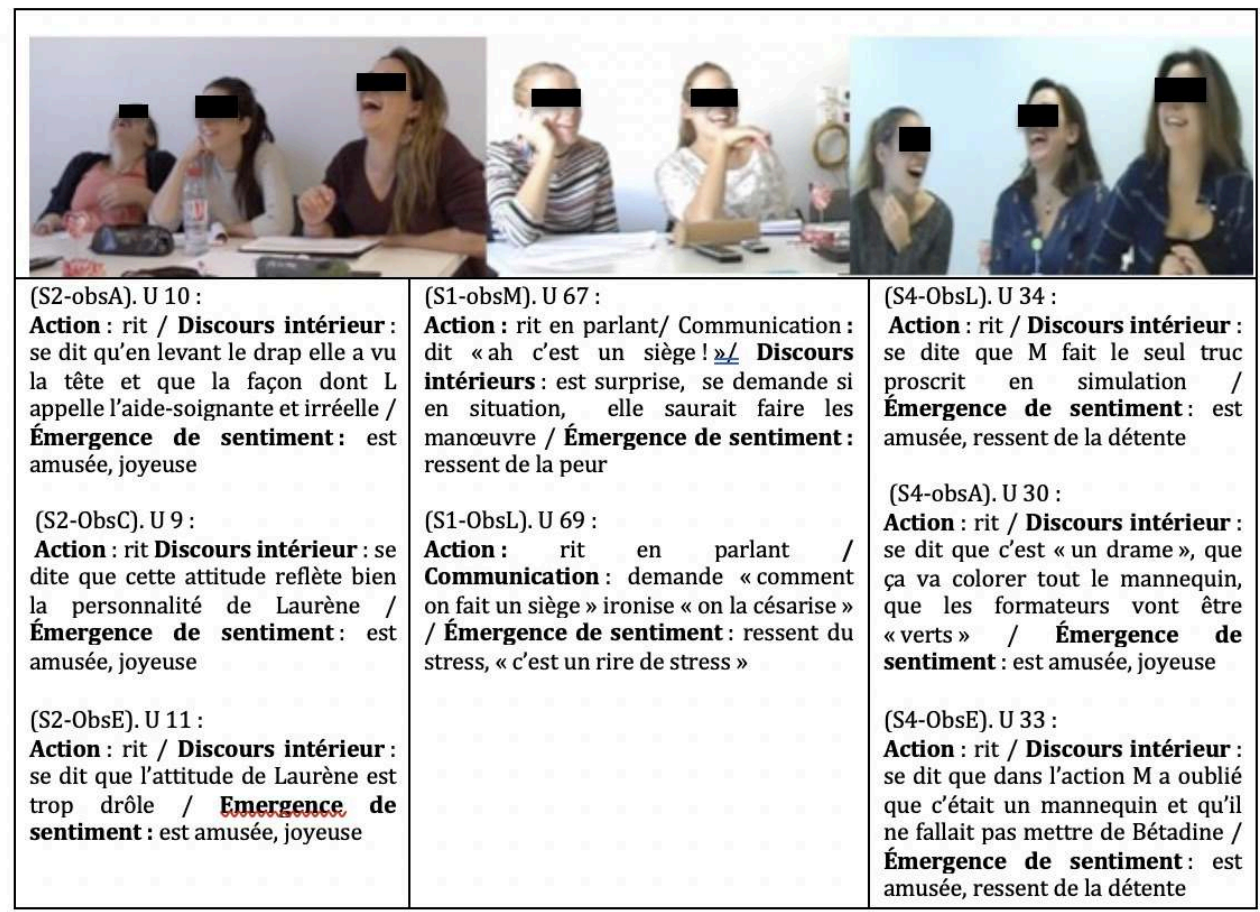

Ce phénomène idiosyncratique, manifeste, par un mouvement du corps sans impression de contrôle, la pensée des observatrices. Il est polymorphe (sourire, éclat de rire, rire contenu), individuel ou collectif. Il traduit trois types de significations de ce qui fait expérience pour les étudiantes à l'instant $t$ :

- le plaisir qui accompagne l'activité d'observation (curiosité quant au scénario dissimulé par les formateurs, ambiance entre observatrices, humour, ironie);

- la satisfaction d'avoir anticipé le bon diagnostic de la situation prévue par les formateurs ;

- un jugement de non-pertinence quant à l'activité observée.

Le rire est l'expression d'une émotion à valence positive (joie, gaité) ou négative (stress, peur, agacement). Certaines étudiantes utilisent l'humour et l'ironie pour susciter une distanciation avec l'activité ressentie comme stressante. Le rire est décrit dans le référentiel (S) des étudiantes comme une détente momentanée, une défense répondant à un conflit émotionnel, un facteur de stress, sans agressivité même s'il concerne un tiers.

(S2-ObsE). S de U 21: [rire] c'est «le partage des difficultés que l'on rencontre aussi », « ce n'est pas de la moquerie ».

(S2-ObsA). S de U 10 : [rire] permet de se détendre.

(S3-ObsM). S de U59: [rire en situation d'observation] permet de «relâcher la pression ".

(S3-obsc). S de U33 : [rire] c'est compatir, « on ne voudrait pas être à leur place mais en même temps c'est drôle de les voir ».

64 Ainsi, le rire participe au jeu de proximité-distance entre les observatrices et les actrices (distanciation des corps) mais aussi à extraire les observatrices de la situation vécue (distanciation des mondes). Il contribue à la dynamique collective de l'activité en 
tant qu'acte social traduisant de la complicité et révèle des jugements tout en atténuant leurs aspects déplaisants.

\section{Discussion}

Tout comme dans les dispositifs de vidéo-formation, l'activité d'observation de situations de SHF, en tant que déséquilibrage-rééquilibrage du couplage observatrices et environnement de formation, consiste :

- en une mise en relation de l'activité visionnée avec une activité autre, propre aux observatrices;

- en des processus de transformations de connaissances relatifs à l'exercice professionnel et au dispositif de formation.

Toutefois, l'émergence de rires et de reproductions de gestes fins de la pratique professionnelle apparait comme une singularité du contexte d'observation étudié. En considérant l'intensité de l'engagement des observateurs dans l'activité (permanence des processus attentionnels), l'immersion fictionnelle (spécifiée par l'émergence de gestes et d'émotions) ainsi que l'occurrence des rires (Bergson, 1900), nous rapprochons l'activité d'observation étudiée de celle de jeu (Caillois, 1958; Henriot, 1983 ; Huizinga, 1938). En effet, selon Henriot, le rire est preuve de jeu mais tout jeu ne se traduit pas par le rire. Le jeu désigne à la fois l'activité (play) spécifique et spécifiée par la totalité nécessaire à son fonctionnement (game). Henriot considère que tout jeu est structure qui n'existe, à titre de jeu, que s'Il est inventé et réinventé, qu'il s'offre à la praxis de quelqu'un qui se définit comme joueur. Le phénomène de jeu a pour primat la signification de jeu accordée par un individu, sur la structure, soit une «situation structurée par des règles dans laquelle le sujet s'oblige à prendre un certain nombre de décisions [...] en fonction d'un contexte plus ou moins aléatoire » (Henriot, 1983, p. 38).

\subsection{Observation de situation de SHF : une pratique de jeu}

Nous reprenons ici les caractéristiques du jeu, définies par Caillois, Huizinga et Henriot et redéfinissons à travers elles, l'activité d'observation comme pratique ludique.

\section{- une activité libre}

Jouer a pour principe fondamental d'être une activité libre et volontaire (Caillois, 1958 ; Henriot, 1983 ; Huizinga, 1938). En SHF, l'observation n'est ni objectivée ni supervisée par les formateurs. Les étudiantes disposent sans contraintes prescriptives, de cet espace de formation. Elles organisent et objectivent l'activité autour d'« en-jeux » consensuellement partagés (découverte, résolution d'un problème obstétrico-pédiatique). Les en-jeux traduisent le primat de l'intrinsèque de l'activité ludique. Ils sont construits dans la dynamique du couplage, guident et organisent l'observation en activité de nature ludique.

\section{- une activité incertaine}

Les activités exploratoires et exécutoires portent la question de la réussite et des risques quant aux en-jeux : quel est le problème de la situation prévu par les formateurs ? Comment va s'opérer sa résolution par les actrices? Vont-elles réussir? Comment les observatrices agiraient ou devraient agir en situation ? Comment va se terminer la situation ? La curiosité et l'engagement dans l'activité d'observation émanent de la part énigmatique de la situation de simulation. Suspens et risques sont les composantes subjectives du jeu avec le désir 
d'éprouver et de s'éprouver. Elle est vécue comme dialectique entre certitudes et incertitudes, les en-jeux portent la nature agonale de l'activité ludique.

\section{- une activité séparée}

L'activité émerge dans une sphère (espace, temps) simulant le réel et suspendant momentanément tout lien avec ce qui en est extérieur. En cela elle est séparée. La salle de débriefing délimite l'espace d'observation comme la situation de simulation délimite sa durée. Ces conditions facilitent l'immersion immédiate et permanente, dans la situation visionnée. L'absorption des observatrices en situation simulée et leur détachement du monde extérieur caractérisent l'expérience de l'état de flow (Csikszentmihalyi, 1990, 2004). Le flow est un état dynamique d'implication totale. Il se manifeste dans tout type d'activité, notamment le jeu, lorsqu'il y a perception d'une adéquation entre les capacités personnelles d'un acteur et le défi ou les exigences de l'activité en cours. Cette expérience est favorisée par le plaisir procuré par l'activité en cours, et la convivialité. Le rire de satisfaction ou de connivence témoigne de cet état et le potentialise. Le rire peut aussi être un moyen de prolonger-restaurer l'état de flow en réduisant le stress ressenti lors de l'activité d'observation. En effet selon Csikszentmihalyi, l'état de flow est caractérisé par une activité portée par un challenge dont le vécu n'est ni ennuyeux ni anxiogène (Csikszentmihalyi, 1990).

\section{- une activité fictive}

Par un jeu de proximité-distance entre activité observée et activité propre, les étudiantes s'immergent fictionnellement en situation. Les observatrices agissent, décident, ressentent des émotions. Comme le postule Henriot, contrairement à Caillois, le jeu n'est pas une évasion en dehors du réel mais s'inscrit dans la réalité matérielle et sociale. Jouer c'est dominer ses rêves et les contrôler. Les observatrices savent ce qu'elles font, savent qu'elles font semblant.

\section{- une activité auto-réglée}

Dans tout jeu règne un ordre spécifique et absolu dont le trait positif est de créer de l'ordre. L'activité d'observation n'est pas encadrée par les formateurs. Ainsi les principes adoptés par les observatrices sont arbitraires, consensuels et organisent l'activité. Nous en relevons deux principaux. La coopération permet d'exploiter avec efficience l'environnement constitué socialement et techniquement, pour majorer leurs capacités à diagnostiquer et solutionner. La bienséance contribue à créer une atmosphère de travail conjuguant plaisir (rires, humour, connivence) et sérieux. Les observatrices pondèrent les jugements critiques envers les actrices, apportent des réponses aux divers questionnements formulés, autorégulent les interactions pour permettre à chacun d'observer.

\section{- une activité improductive ?}

Si l'observation ne laisse aucun résultat tangible comme l'avance Caillois, elle s'accompagne de transformations relatives au dispositif de formation et aux potentialités du simulateur qui permettent de mieux anticiper ce qui va se jouer et d'avoir la main sur le scénario. Ces transformations révèlent, comme le postule Henriot, que le jeu modifie les qualités et aptitudes du joueur. Il développe l'adresse physique et mentale. Le joueur est l'œuvre de son jeu. De l'observation émergent des transformations, renforcements, créations de connaissances obstétrico-pédiatiques théoriques, techniques, procédurales et des connaissances sur soi. L'observation porte sur des situations d'urgences rares, pour lesquels les risques médico-légaux sont majeurs en situation réelle. L'activité d'observation présente un intérêt transformatif éducatif tout en euphémisant les risques pour les participantes. Ainsi l'activité d'observation comporte une fonction utilitaire pour la pratique maïeutique et une fonction ludique pour la conduite de l'activité. Nous la qualifions de jeu-sérieux. Le jeu- 
sérieux est un objet dont la finalité première est autre que le divertissement (Michael \& Chen, 2006). Il mélange "une dimension sérieuse, renvoyant à tout type de finalité utilitaire, et une dimension ludique, correspondant à un jeu matérialisé sur tout type de support» (Djaouti, 2011, p. 22). spécifiquement de jeu-utilitaire. Les activités individuelles des observatrices s'organisent collectivement par construction commune d'invariants significatifs et structurels et par leurs ajustements mutuels. Ils participent à apporter une réponse satisfaisante aux enjeux d'ordre strictement conventionnel et caractérisent le jeu d'une nature agonale.

\subsection{Ludicisation de l'espace de formation}

69

Henriot adopte une approche phénoménologique du jeu permettant de saisir ce phénomène comme conduite significative d'une attitude et d'un mode d'être (Henriot, 1983). Il postule que le phénomène jeu appartient au domaine du signifiant et qu'une situation ne peut être définie au préalable comme jeu. Pour jouer, il faut se projeter et s'inventer dans la production de son acte. Ce primat de l'intrinsèque du jeu est révélé par le fait que les étudiantes déploient une activité de nature ludique alors que l'observation n'est pas structurée par des règles traçant un schéma d'une conduite hypothétique par les formateurs. Les observatrices adoptent une attitude ludique $e^{12}$ participant à un processus de contagion ludique de l'environnement d'observation. L'attitude ludique permet de considérer l'environnement dans sa dimension de jouabilité, dans sa (re)création (Brougère, 2013), sa ludicisation (Genvo, 2015). La ludicisation traduit le processus appropriation de l'environnement d'observation (spatial, social, technique) dans le monde, le corps et la culture propre de chaque étudiante (Theureau, 2011). L'appropriation définit un processus de transformations majorantes émergeant du couplage asymétrique, par lequel les étudiantes adoptent et adaptent l'environnement d'observation et l'inscrivent dans leur pratique de formation (Poizat, 2014).

\section{- Intégration au monde propre}

Elle consiste à attribuer au dispositif une signification de ressources permettant de connecter les expériences simulées avec les expériences propres (immersion, résonance). Les incertitudes et incomplétudes de la situation sont interprétées comme des en-jeux et l'environnement d'observation est appréhendé dans sa dimension de jouabilité et de créativité.

- Intégration au corps propre

Elle prolonge l'intégration de l'environnement du monde propre au corps propre. Elle consiste à incorporer les ressources du dispositif comme prolongement du corps dans une logique d'opérationnalité. L'activité allouée d'une nature agonale vise l'agir performant pour surmonter les défis établis arbitrairement par les observatrices en situation. L'activité se caractérise par la duplicité des observatrices comme oscillation constante du dehors au dedans, de l'agir pour soi et l'agir à travers un autre. L'attitude ludique a pour ancrage la «feintise ludique partagée» (Schaeffer, 1999) : elles font «comme si » la situation était réelle, agissent (miment), expérimentent, éprouvent comme si elles y étaient.

- Intégration à la culture propre

Elle consiste en la construction d'une culture d'action partagée ludique permettant 
d'organiser l'activité coopérativement pour gagner en efficacité. Le jeu s'organise autour de constructions de principes (individuels et consensuels) qui guident l'activité et circonscrivent «l'arbitraire ludique» (Henriot, 1983). L'arbitraire ludique réfère à l'ensemble de procédés qui permettent de réaliser des décisions dans un contexte qui les guide. Il traduit le fait que le jeu n'existe que par la volonté du joueur (Henriot, 1983). Ainsi l'in-culturation du dispositif s'accompagne de la transformation de l'environnement de formation des observatrices en "mondes ludiques $»^{13}$ possibles (Ter Minassian, Rufat, Coavoux, \& Berry, 2011). Ces mondes (situation d'observation, situation simulée en cours, passée, situation vécue rejouée) offrent l'opportunité d'expériences nouvelles tout en les contraignant. Ils participent à des processus de transformations relatifs à l'exercice de la maïeutique (fonction utilitaire) mais aussi relatifs aux principes de conception des cessions de simulation (modalités ludiques, jouabilité) dont émanent des processus créatifs conciliant jeu et apprentissage-développement. Cette in-culturation contribue à la spécification de futurs possibles en stage (pratique clinique) et en simulation (scénarios projetés, pratique imaginée et simulée).

La ludicisation, entendue comme appropriation générique ludique de l'environnement de visionnage dans les mondes, corps, cultures des observatrices mais aussi son potentiel d'apprentissage-développement, permettent de le définir comme une sphère vidéo-ludoutilitaire du dispositif de formation. Elle s'apparente aux dispositifs de vidéo-formation par alloconfrontation collective de paires en situations professionnelles avec pour particularités d'être simulées, de reproduire des évènements critiques rares et d'être contraintes par la dimension temporelle. La ludicisation consiste à considérer certaines perturbations du coupage structurel comme les agonistes du jeu (en-jeux) et à organiser l'activité collectivement. Son potentiel transformatif est majorant via des processus créatifs rejoués, ici et maintenant, mais aussi projetés dans le futur en tant qu'actions jugées prometteuses de performance en situations similaires.

\section{Conclusion}

L'analyse de l'activité d'observation a permis comprendre comment elle s'organise en jeu de nature sérieuse et agonale. Si la dynamique de l'activité individuelle-collective participe à un processus d'apprentissage-développement majorant, elle s'accompagne également de constructions erronées ou de mises en évidence de connaissances instables. Ainsi l'environnement d'observation, qualifié de vidéo-ludo-utilitaire, participe à déclencher des transformations désirables mais devrait aussi accompagner les processus de remédiations, renforcements et de transférabilité. Le débriefing pourrait accompagner ces processus de typicalisation assurant une généralité à l'action située, en considérant les expériences simulées des actrices et celles des observatrices. Dans une visée praxéologique, ces résultats nous amènent à repenser plus globalement la conception d'une session de formation par SHF à trois niveaux. Premièrement, en constituant des groupes d'observatrices (effectif et niveau de formation) rendant viable et relativement stable le niveau collectif de structuration et de signification de l'activité émergeant des activités individuelles et de leurs articulations locales (Theureau, 2006). Deuxièmement, en considérant les composantes fictionnelles et ludiques qui émanent de l'expérience comme débrayage entre réel et « comme si » (Durand, 2008). Elles sont propices à l'engagement permanent dans l'activité d'observation et sources de motivation, plaisir, transformations. Troisièmement, en revisitant la conduite du débriefing pour décaler sa focale de l'activité simulée à l'ensemble des activités qui y 
sont déployées et accompagner l'ensemble des transformations. Enfin, nous nous interrogeons sur une possible contagion ludique de l'activité simulée dans un dispositif proposant une alternance d'observations et de mises en situation simulées. Cette possibilité irait à l'encontre des préconisations de la HAS $^{14}$ (HAS, 2012b). Pourquoi envisager la simulation comme jeu « serait une perte de contrôle pour une formation réussie?» La négation du jeu est présentée comme un principe de précaution sécurisant le vécu des participants. Mais peut-on maitriser que la simulation ne comporte pas de composante ludique, alors que le jeu (game) ne peut présumer du jouer (play) ? Sans écarter que la finalité première de la simulation est utilitaire, nous interrogeons cette conception antithétique simulation-jeu comme garante d'une formation au potentiel fécond.

\section{BIBLIOGRAPHIE}

Bergson, H. (1900). Le rire. Paris : PUF.

Boet, S., Granry, J.-C., \& Savoldelli, G. (2013). La simulation en santé: de la théorie à la pratique. Paris : Springer-Verlag.

Brandom, R. (1994). Making it explicit: Reasoning, representing, and discursive commitment. Cambridge, Mass : Harvard University Press.

Brougère, G. (2013). Jacques Henriot et les sciences du jeu ou la pensée de Villetaneuse. Sciences du jeu, (1). http://sdj.revues.org/202

Caillois, R. (1958). Les jeux et les hommes : le masque et le vertige. Paris : Gallimard.

Csikszentmihalyi, M. (1990). Flow: The psychology of optimal experience. New York : Harper Row.

Csikszentmihalyi, M. (2004). Vivre : la psychologie du bonheur. Paris : Pocket.

DGOS. (2013). Instruction DGOS/PF2 n 2013-383 du 19 novembre 2013 relative au développement de la simulation en santé, Pub. L.

Dieckmann, P., Molin Friis, S., Lippert, A., \& Østergaard, D. (2009). The art and science of debriefing in simulation: Ideal and practice. Medical Teacher, 31(7), 287-294.

Dieumegard, G. (2009). Connaissances et cours d'expérience vers une grammaire minimale de description dans les situations d'éducation et de formation. Revue d'anthropologie des connaissances, 3(2), 295-315. https://doi.org/10.3917/rac.007.0295

Djaouti, D. (2011). Serious Game Design Considérations théoriques et techniques sur la création de jeux vidéo à vocation utilitaire. Thèse en informatique, Université de Toulouse III Paul Sabatier, Toulouse.

Durand, M. (2008). Un programme de recherche technologique en formation des adultes : Une approche enactive de l'activité humaine et l'accompagnement de son apprentissage/ développement. Éducation et didactique, 2(3), 97-121. https://doi.org/10.4000/ educationdidactique. 373 
Esfeld, M. (2001). La normativité sociale du contenu conceptuel. In Dossier : Normativité. https:// doi.org/10.4000/books.editions

Flandin, S., Leblanc, S., \& Muller, A. (2015). Vidéoformation « orientée activité » : Quelles utilisations pour quels effets sur les enseignants. Analyse du travail et formation dans les métiers de l'éducation, Raisons éducatives, 19, 179-198.

Flandin, S., \& Ria, L. (2018). Qu'apprennent les enseignants novices de l'observation de leurs pairs et comment l'apprennent ils? Une étude de cas. In C. Gaudin, S. Flandin, S. Moussay, \& Sébastien Chaliès (Eds.), Vidéo-formation et développement de l'activité professionnelle enseignante (pp. 49-64). Paris : L'Harmattan.

Gaudin, C., \& Chaliès, S. (2012). L'utilisation de la vidéo dans la formation professionnelle des enseignants novices. Revue française de pédagogie, 178, 115-130.

Gaudin, C., Flandin, S., Moussay, S., \& Chalies, S. (2018). Vidéo-formation et développement de l'activité professionnelle enseignante. Paris : L'Harmattan.

Gaudin, C., Flandin, S., Ria, L., \& Chaliès, S. (2016). An exploratory study of the influence of video viewing on preservice teachers' teaching activity: normative versus developmental approaches. Form@re, 14(2), 21-50.

Genvo, S. (2015). Penser les phénomènes de ludicisation à partir de Jacques Henriot. Sciences du jeu, 1. http://journals.openedition.org/sdj/251

Harder, N., Ross, C. J. M., \& Paul, P. (2013). Student Perspective of Roles Assignment in HighFidelity Simulation: An Ethnographic Study. Clinical Simulation in Nursing, 9(9), 329-334.

HAS. (2012a). Guide de bonnes pratiques en matière de simulation en santé. https://www.has-sante.fr/ portail/upload/docs/application/pdf/2013

HAS. (2012b). Rapport de mission : État de l'art (national et international) en matière de pratiques de simulation dans le domaine de la santé. https://www.has-sante.fr/portail/upload/docs/application/ pdf/2012-01/simulation_en_sante_-_rapport.pdf

HAS. (2017). Simulation en santé. Fiche DPC. https://www.has-sante.fr/portail/jcms/c_2807140/fr/ simulation-en-sante

Henriot, J. (1983). Le jeu. Paris : Synonyme-S.O.R.

Hober, C., \& Bonnel, W. (2014). Student Perceptions of the Observer Role in High-Fidelity Simulation. Clinical Simulation in Nursing, 10(10), 507-514.

Horcik, Z., \& Durand, M. (2015). L'expérience mimétique dans l'apprentissage adulte : le cas des formations par simulation. Revue suisse des sciences de l'éducation. 1(37), 167-186.

Huizinga, J. (1938). Homo ludens : essai sur la fonction sociale du jeu. Paris : Gallimard.

Jaffrelot, M., Boet, S., Di Cioccio, A., Michinov, E., \& Chiniara, G. (2013). Simulation et gestion de crise. Réanimation. 22(6), 569-576.

LeBel, M.-E., Haverstock, J., Cristancho, S., van Eimeren, L., \& Buckingham, G. (2018). Observational Learning During Simulation-Based Training in Arthroscopy: Is It Useful to Novices? Journal of Surgical Education, 75(1), 222-230.

Leblanc, S. (2012). Conception d'environnements vidéo numériques de formation. Développement d'un programme de recherche technologique centré sur l'activité dans le domaine de l'éducation. Note de synthèse pour l'Habilitation à Diriger les Recherches. Université Montpellier 3. 
Leblanc, S. (2014a). Expériences mimétiques en vidéoformation et transformations de l'activité professionnelle. Recherche \& formation, 75, 37-50. https://doi.org/10.4000/rechercheformation. 2169

Leblanc, S. (2014b). Vidéo formation et transformations de l'activité professionnelle. Activités, 11(2). https://doi.org/10.4000/activites.968

Leblanc, S. (2015). Usages de la vidéo au sein d'un processus progressif de professionnalisation de la formation des enseignants. In L. Ria (Ed.), Former les enseignants au $21^{e}$ siècle. Etablissement formateur et vidéo formation (pp. 203-214). Bruxelles : De Boeck.

Leblanc, S. (2018). Analysis of Video-Based Training Approaches and Professional Development. Contemporary Issues in Technology and Teacher Education, 18(1), 125-148.

Maturana, H. R., \& Varela, F. J. (1994). L'arbre de la connaissance. Paris : Addison-Wesley.

Michael, D., \& Chen, S. (2006). Serious games: Games that educate, train and inform. Boston, Mass: Thomson Course Technology.

Norman, D. (1993). Artefacts cognitifs. In B. Conein, N. Dodier, \& L. Thévenot (Eds.), Les Objets dans l'action (pp 15-34). Paris : Éditions de l'Ecole des hautes études de sciences sociales.

O'Regan, S., Molloy, E., Watterson, L., \& Nestel, D. (2016). Observer roles that optimise learning in healthcare simulation education: A systematic review. Advances in Simulation, 1(1). https:// doi.org/10.1186/s41077-015-0004-8

Pierce, C. S. (1931). The Collected Papers of Charles Sanders Peirce. Cambridge : Harvard University Press.

Poizat, G. (2014). Le concept d'appropriation en formation des adultes : polysémie théorique et diversité pratique. In J. Friedrich \& J. C. Pita Castro (Eds.), Recherches en formation des adultes : un dialogue entre concepts et réalité (pp 40-68). Dijon : Raison et passions.

Rabardel, P. (1995). Les hommes et les technologies; approche cognitive des instruments contemporains. Paris : Armand Colin.

Ria, L., \& Leblanc, S. (2011). Conception de la plateforme de formation Néopass@ction à partir d'un observatoire de l'activité des enseignants débutants : Enjeux et processus. Activités, 8(2). https://doi.org/10.4000/activites.2618

Ria, L., Serres, G., \& Leblanc, S. (2010). De l'observation vidéo à l'observation in situ du travail enseignant en milieu difficile : étude des effets sur des professeurs stagiaires. Schweizerische Zeitschrift für Bildungswissenschaften, 32(1), 105-120.

Roche, L., \& Gal-Petitfaux, N. (2012). La médiation audio-visuelle pour former à l'expérience corporelle de l'enseignant d'EPS en situation de classe. Staps, 98(4), 95.

Sartre, J.-P. (1943). L'être et le néant. Paris : Gallimard.

Saury, J. (2008). La coopération dans les situations d'intervention, de performance et d'apprentissage en contexte sportif. Contribution au développement d'un programme de recherche en ergonomie cognitive des situations sportives en STAPS. Note de synthèse (non publiée) pour l'habilitation à diriger des recherches, université de Nantes.

Schaeffer, J.-M. (1999). Pourquoi la fiction ? Paris : Seuil

Ter Minassian, H., Rufat, S., Coavoux, S., \& Berry, V. (2011). Comment trouver son chemin dans les jeux vidéo ? Pratiques et représentations spatiales des joueurs. Espace géographique, 40(3), 245-262. 
Theureau, J. (2004). Le cours d'action: Méthode élémentaire. Toulouse : Octarès.

Theureau, J. (2006). Le cours d'action: Méthode développée. Toulouse : Octarès.

Theureau, J. (2011). Appropriations 1, 2 \& 3 ou Un exemple de pouvoir heuristique et de capacité de croissance d'un programme de recherche ou Appropriation, Incorporation \& In-culturation. Journée Ergo-Idf. Paris.

Theureau, Jacques. (2015). Le cours d'action : L'enaction \& l'expérience. Toulouse : Octarès.

Varela, F. J. (1989). Autonomie et connaissance-essai sur le vivant. Paris : Seuil.

Varela, F. J., Thompson, E., Rosch, E., \& Havelange, V. (1993). L'inscription corporelle de l'esprit : sciences cognitives et expérience humaine. Paris : Seuil.

Wulf, C. (2007). Une anthropologie historique et culturelle rituels, mimésis sociale et performativité. Paris : Téraèdre.

Zottamnn, J. M., Dieckermann, P., Taraswzow, T., Rall, M., \& Fischer, F. (2018). Just watching is not enough: Fostering simulation-based learning with collaboration scripts. GSM Journal for Medical Education, 35(3).

\section{ANNEXES}

\section{Annexe 1 : Identification d'une séquence}

Tableau 7 : Illustration d'une séquence (S5-ObsA) Chart 7 : Illustration of a sequence (S5-ObsA)

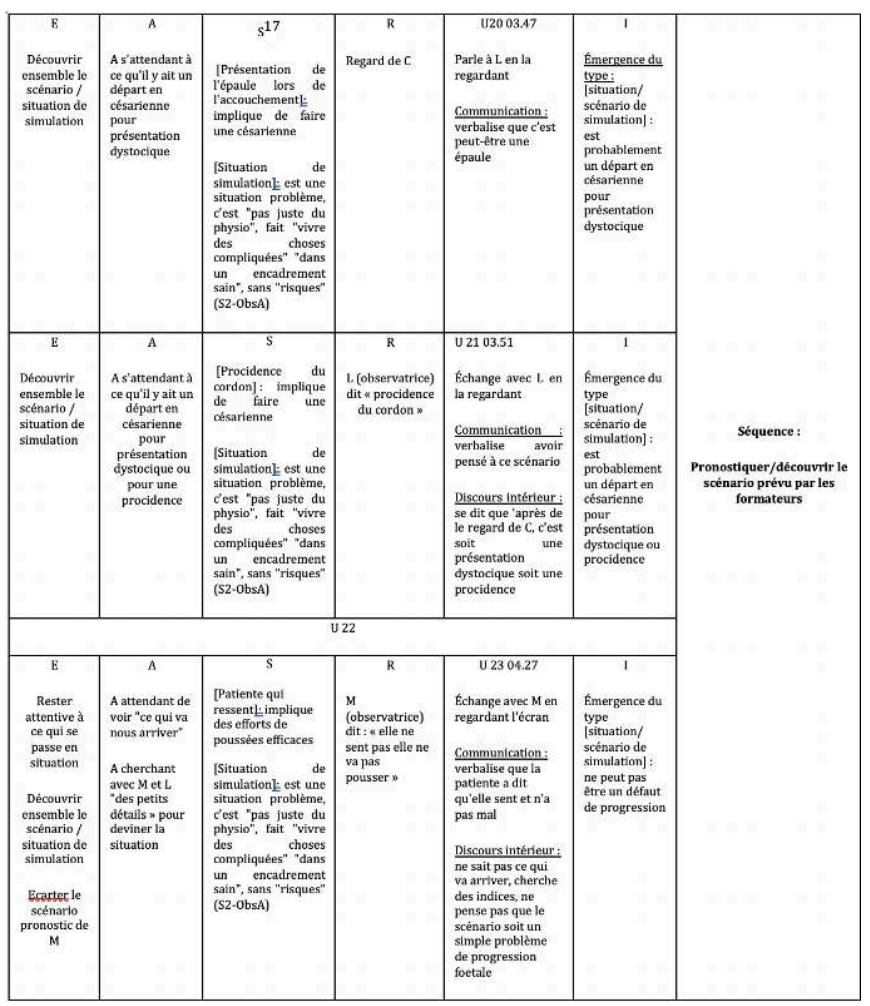




\section{Annexe 2}

La modélisation a été réalisée sous forme de tableau à double entrée. Chaque signe est reporté sur l'axe horizontal et identifié par un numéro restituant la chronologie de leur émergence dans le cours d'expérience. Les structures significatives de différents rangs ont été placées dans l'axe vertical. Les séquences et macro-séquences sont indexées d'une couleur. Leur émergence dans un signe est identifiée par un codage couleur de la case ayant pour entrée le numéro du signe et la dénomination de la structure significative. La modélisation étant longue (90 signes), elle est présentée élaguée de signes 1 à 8 et de 33 à 77.

Tableau 8 : Extrait d'une modélisation de cours d'expérience avant diagnostic de pathologie. Chart 8 : Extract from a course-of-experience modelling before pathology diagnosis

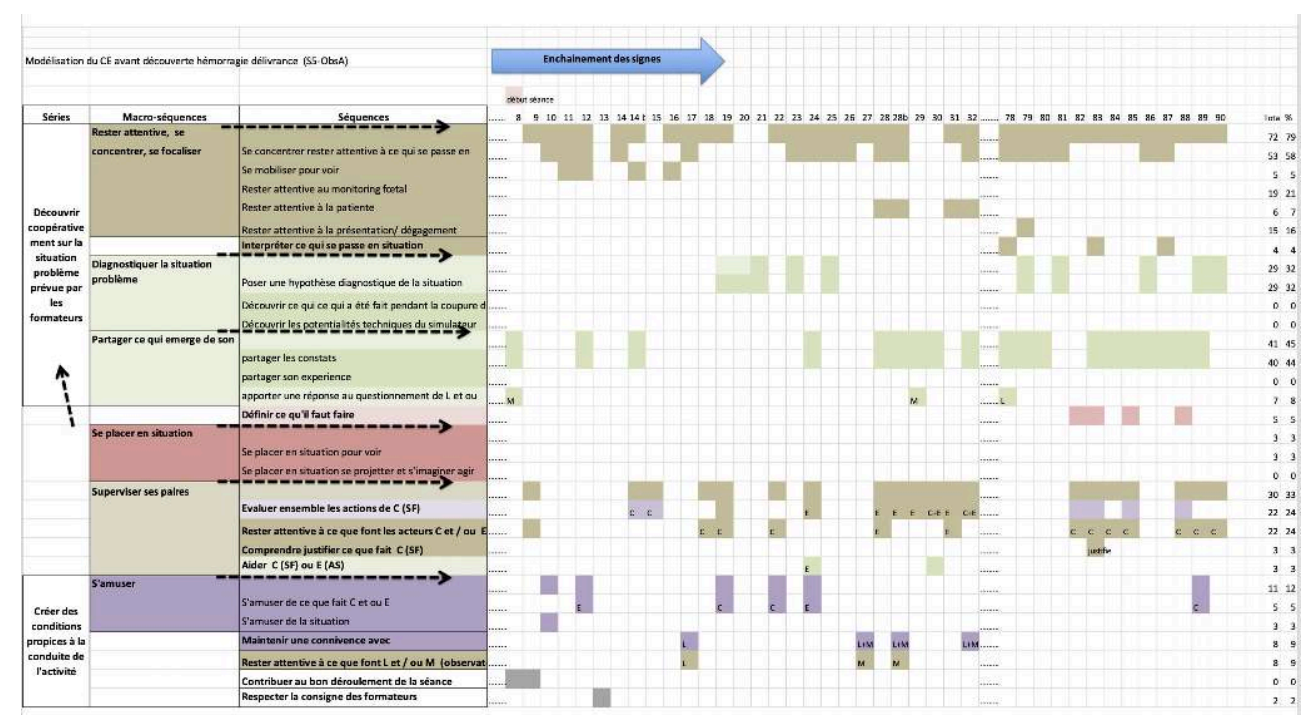

\section{Annexe 3}


Tableau 9 : Extrait d'une modélisation de cours d'expérience après diagnostic de pathologie. Chart 9 : Extract from a course-of-experience modelling after pathology diagnosis

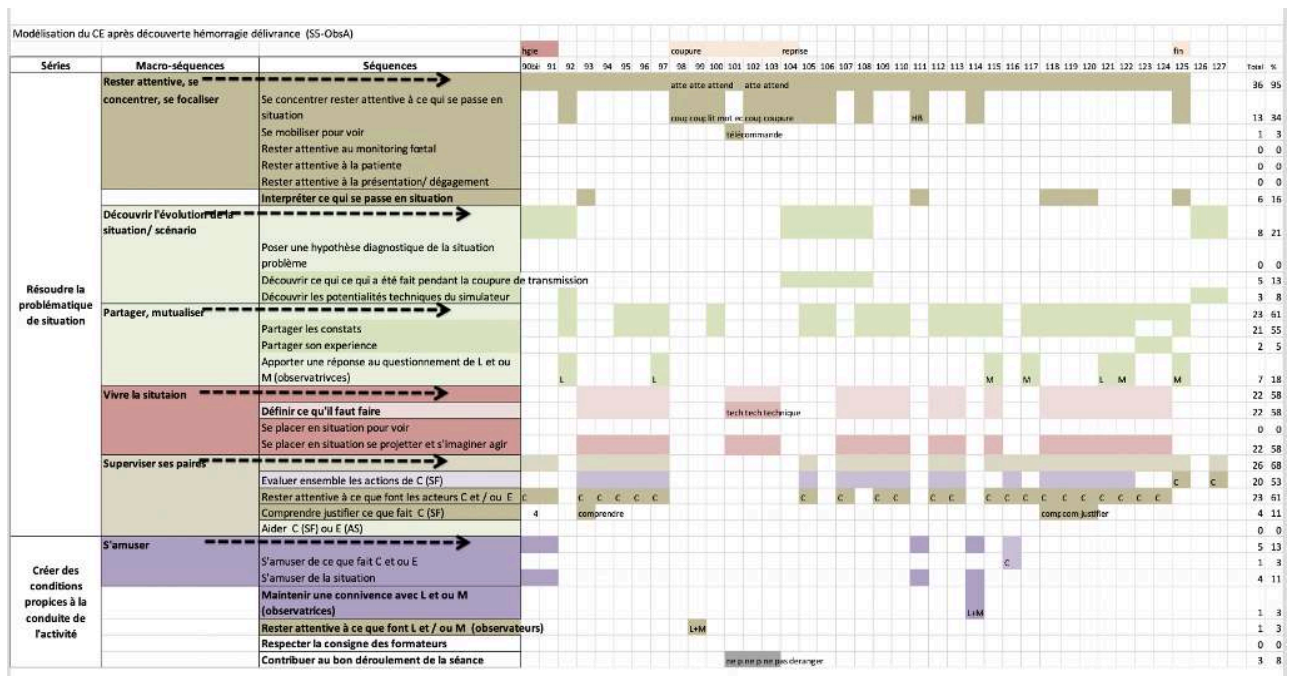

\section{NOTES}

1. La fidélité réfère au degré avec lequel la simulation imite la réalité. Selon de degré de réalisme, les techniques de simulation seront qualifiées de basse ou haute-fidélité (HAS, 2012a, p. 12)

2. Un artefact est une ressource offerte par l'environnement et mobilisée par un acteur pour structurer, exécuter, optimiser ses actions (Norman, 1993). Les artefacts sont inscrits dans les lieux, dispositifs techniques, objets, individus, ils secondent les actions en tant qu'offres et opportunités.

3. Le formateur explique aux apprenants, mais également aux observateurs, le déroulement de la séance de simulation et les consignes pour l'optimiser (HAS, 2012a, p. 14). Afin d'observer sans perturber les apprenants, et pouvoir analyser les actions réalisées avec le groupe, les séquences sont retransmises en direct dans une autre salle (HAS, 2012a, p. 66)

4. Selon cette approche, qui guide la recherche en médecine, les études sont effectuées de manière systématique en double aveugle et mettant en jeu des groupes expérimentaux et des groupes de contrôles et des pré-tests et post-tests

5. L'alloconfrontation consiste à proposer via des images animées, une représentation observable d'une situation professionnelle de référence permettant a) de découvrir les multiples dimensions qui la composent b) de confronter ses propres intentionnalités aux faits observés (principe d'autoréférence) et /ou confrontations d'opinions c) de reconstruire les pratiques par un réglage réciproque théorie-pratique lors du feed-back (principe de régulation).

6. Tout système est autonome, opérationnellement clos et son couplage à l'environnement est asymétrique (Maturana \& Varela, 1994 ; Varela, 1989).

7. Nous nommerons observation, l'activité menée par les participantes «non actrices" des situations simulées comprenant l'observation et toute activité afférente, que nous ne pouvons présupposer mais qui participe à son flux continu.

8. Ensemble des phénomènes mécaniques et physiologiques qui ont pour conséquence l'expulsion fœtale.

9. Nous nous appuyons sur une définition «minimale » de la coopération (Saury, 2008, p. 35) qui met en évidence « trois caractéristiques principales, étroitement liées : (a) l'interdépendance des activités individuelles, (b) le partage - au moins potentiel - d'une situation de travail, et (c) l'articulation collective in situ et en temps réel d'activités individuelles autonomes ». 
10. Dispositif permettant d'optimiser la ventilation en maitrisant les pressions et en évitant les collapsus alvéolaires.

11. Manœuvre obstétricale de réduction de la dystocie des épaules, préconisée lorsque les deux épaules sont au-dessus du détroit supérieur.

12. Henriot définit trois caractéristiques au « jouer » comme attitude : a) l'incertitude qui serait le caractère le plus apparent de toute conduite ludique, b) la duplicité comme capacité à se «dédoubler » en mettant une certaine distance entre soi et le jeu, c) l'illusion qui fait que le jeu peut se déployer (Henriot, 1983, p. 83)

13. Un monde ludique réfère à tout espace qui n'est pas un espace de pratique ludique mais lié à la culture ludique des acteurs. Cette culture ludique participe à l'émergence et la structuration de communautés de pratiques (Ter Minassian, Rufat, Coavoux, \& Berry, 2011).

14. «La simulation professionnelle n'est pas un jeu. Elle donne à voir les compétences, les échecs, questionne l'ego des professionnels, et peut susciter une émotion réelle des participants. Ces dimensions peuvent servir utilement l'acquisition des compétences, mais leur gradation doit être particulièrement contrôlée, et probablement appliquée avec discernement selon les personnalités des opérateurs. Car l'opérateur de conduite en formation est bien la cible du dispositif ; et c'est bien aussi le seul élément du puzzle qui ne soit pas simulé » (HAS, 2012b, p. 26)

\section{RÉSUMÉS}

L'objectif de cette contribution est de produire des connaissances sur l'activité d'observation lors de séance de simulation haute-fidélité (SHF), en formation initiale de la maïeutique. Cette activité reste une zone d'ombre à la fois du point de vue scientifique et également du point de vue de la formation, alors qu'il s'agit d'une pratique ordinaire en formation initiale des métiers de la santé. L'observation permet de répondre à la contrainte de former un effectif conséquent d'étudiant·e·s à travers un dispositif pédagogique qui ne soit pas trop chronophage tout en tirant profit des équipements vidéo, des salles de SHF, tels qu'ils sont préconisés par la haute autorité de santé (HAS). Cette étude se place dans le cadre théorique du cours d'action (Theureau, 2004, 2006) pour analyser l'activité d'observation de cinq étudiantes sages-femmes, à l'occasion d'un module de plusieurs séances de SHF. Ce cadre épistémologique permet de prendre en compte le point de vue des observatrices pour accéder à la compréhension de leur activité, en partie silencieuse, ainsi qu'à son potentiel transformatif. Les analyses des données construites à partir des vidéos des situations d'observation et des entretiens d'autoconfrontations, montrent que l'activité d'observation a) s'organise collégialement en conduite de jeu, en considérant les indéterminations de la situation comme en-jeu et b) qu'elle s'accompagne de processus transformatifs majorant et/ou projetés, relatifs à l'exercice professionnel, mais aussi spécifiques à la simulation. Ainsi l'appropriation générique ludique de l'environnement de visionnage et son potentiel d'apprentissage-développement, permettent de le définir comme une sphère vidéoludo-utilitaire du dispositif de formation.

The aim of this contribution is to produce knowledge on observation activity during a highfidelity simulation session (HFS) as part of initial maieutics training. This activity remains a gray area both from a scientific point of view and from the point of view of training, whereas it is standard practice during initial health professions training. Observation makes it possible to respond to the constraint of training a large number of students, by using a pedagogical device 
that is not too time-consuming and which takes advantage of the video equipment and SHF rooms, in accordance with the French high authority of health (HFS)) recommendations. This study falls within the theoretical framework of 'course of action' (Theureau, 2004, 2006), in order to analyze the observation activity of five midwife students, during a module of several HFS sessions. This epistemological framework makes it possible to consider the observers' point of view in order to gain a better understanding of their activity, partly silent, as well as its transformative potential. Data analysis constructed from the videos of the observation situations and self-confrontation interviews show that the observation activity a) is collegially organized as if it were a game, with the indeterminations of the situation being considered as a stake and $b$ ) is accompanied by major and/or projected transformative processes which relate to professional practice and which are also specific to simulation. The playful generic appropriation of the viewing environment and its potential for learning-development thus make it possible to define it as a fun and utilitarian video sphere of the training program.

\section{INDEX}

Keywords : observation, high fidelity simulation, video-training, initial education, midwifery, game

Mots-clés : observation, simulation haute-fidélité, vidéo-formation, formation initiale, maïeutique, jeu

\section{AUTEURS}

\section{HÉLÈNE BOUCHOT}

Département de Maïeutique Site de Nîmes, UFR de Médecine, Université Montpellier. 186 chemin du Carreau de Lanes. CS 83021 - 30908 Nîmes cedex 2. Helene.bouchot@umontpellier.fr

\section{SERGE LEBLANC}

LIRDEF, Université Montpellier et Université Paul Valéry, Faculté d'éducation, Montpellier. ESPE, de l'académie de Montpellier. FDE. 2 place Marcel Godechot. BP 4152. 34092 Montpellier Cedex 5. serge.leblanc@umontpellier.fr 